\title{
Synthesis of substituted-3-iodo-1H-pyrazole derivatives and their further modification under Sonogashira cross-coupling reaction conditions
}

\author{
Rita Mazeikaite, Jurgis Sudzius, Gintaras Urbelis, and Linas Labanauskas* \\ Center for Physical Sciences and Technology, Akademijos 7, LT-08412 Vilnius, Lithuania \\ E-mail: linas.labanauskas@ftmc.lt
}

DOI: http://dx.doi.org/10.3998/ark.5550190.p008.842

\begin{abstract}
A convenient synthetic route for preparation of valuable synthetic intermediates - 1-(1ethoxyethyl)-3-iodo- $1 H$-pyrazole derivatives has been developed. During this work protection reaction of $\mathrm{N}-\mathrm{H}$ bond in substituted 3-iodo-1H-pyrazole derivatives with ethyl vinyl ether and migration of ethoxyethyl protecting group was investigated. Synthetic possibilities of Sonogashira cross-coupling reactions of substituted 1-(1-ethoxyethyl)-3-iodo- $H$-pyrazole derivatives with phenylacetylene were studied and evaluated.
\end{abstract}

Keywords: Sonogashira cross-coupling, 1-(1-ethoxyethyl)-3-iodo-1H-pyrazole, ethoxyethyl protecting group migration

\section{Introduction}

Pyrazole scaffolds can be found in a number of small molecules possessing biological activity. ${ }^{1,2}$ Also it is used in design of new OLED materials. ${ }^{3}$ Substituted pyrazole derivatives may be used as ligands for transition metal-catalyzed reactions. ${ }^{4}$ Moreover, they are very useful synthetic intermediates for other heterocyclic systems such as substituted bis(pyrazolo[4,3d] $[1,2]$ diazepinones, ${ }^{5}$ substituted octahydro- $1 H$-benzo[ $\left.g\right]$ indazole derivatives, ${ }^{6}$ substituted $1 H$ pyrazolo[3,4-c]pyridines ${ }^{7}$ and other.

Electrophilic substitution in the pyrazole ring usually occurs in the 4-th position. Thus, one of the most common ways for the synthesis of 3-substituted pyrazole derivatives is based on the formation of pyrazole ring via condensation of functionalized 1,3-dicarbonyl compounds with hydrazines. ${ }^{8}$ Such methods usually generate mixture of regioisomers and cause problems with isolation of products. In addition, only limited selection of dicarbonyl compounds are readily available. Another pathway for the synthesis of pyrazole derivatives with substituents in 3,4-, 4,5or 3,5-th positions involves palladium catalyzed cross-coupling reactions. ${ }^{9-11}$ Despite the fact that 
number of reports on successful application of cross-coupling in pyrazole chemistry increases, this field is not yet fully investigated.

2-Nitro- and 2-formylhetarylacetylenes are valuable intermediates of new heterosystems. ${ }^{12-15}$ Previous studies of intramolecular cyclisation or cycloisomerisation of 2-formylhetarylacetylenes has showed that the structure of products can vary depending on the nature of used materials. ${ }^{12,16}$ For further studies of cyclisation reactions 1-(1-ethoxyethyl)-3-(phenylethynyl)-1H-pyrazole-4carbaldehyde and 1-(1-ethoxyethyl)-3-nitro-4-(phenylethynyl)-1H-pyrazole were synthesized. Herewith the Sonogashira cross-coupling reaction of 1-(1-protected)-3-iodo-1H-pyrazole derivatives with phenylacethylene was investigated and applied for the synthesis of $\mathrm{N}$-unprotected pyrazoles.

\section{Results and discussion}

As pyrazoles themselves are known as ligands for transition metals, ${ }^{4}$ protection of N-H group of 3iodo- $1 \mathrm{H}$-pyrazole derivatives were necessary in our investigations of cross-coupling reactions. $1 \mathrm{H}$ Pyrazoles 1-5 were protected using Boc anhydride and ethyl vinyl ether. N-ethoxyethyl (EtOEt) and N-Boc protected pyrazole derivatives (1a-5a and 1b-5b, respectively) were synthesized in good to excellent yields (Scheme 1) and used in reactions with lithium organic compounds and Sonogashira cross-coupling reactions. Unfortunately, Boc protecting group was not stable enough both in reactions with lithium organic compounds and during GC-MS analysis. Thus we have decided to focus our further experiments on EtOEt protected pyrazole derivatives.

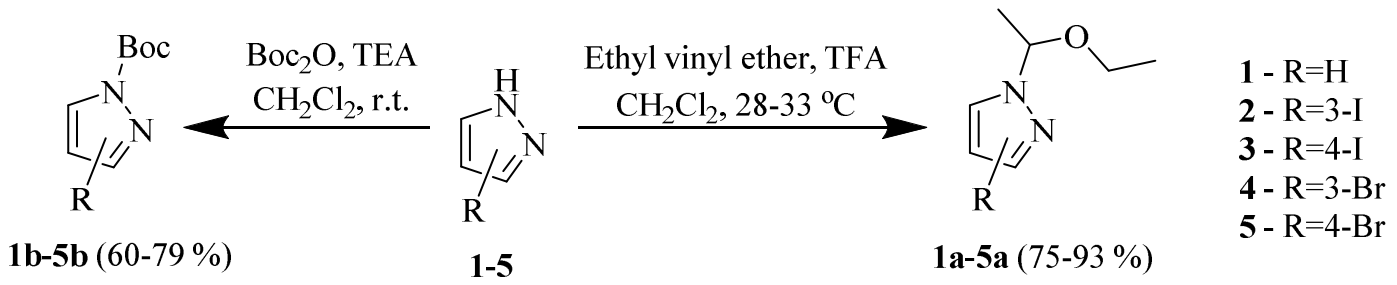

Scheme 1. Protection of free N-H group in 1H-pyrazole derivatives 1-5.

According to literature reports, EtOEt protecting group can be easily introduced and removed from pyrazole ring in mild acidic conditions. ${ }^{17-19}$ Tetrahydropyran (THP) group is recommended for the synthesis of unsymmetrical pyrazole derivatives. ${ }^{20}$ Usually protection reaction of pyrazole ring with EtOEt or THP groups are performed by heating reaction mixture of starting pyrazoles with ethyl vinyl ether or dihydropyran and catalytic amount acid $40-50{ }^{\circ} \mathrm{C}$. Deprotection in acidic conditions is reversible and the removal of ethyl vinyl ether or 3,4-dihydro- $2 \mathrm{H}$-pyran is necessary to complete this reaction. Due to lower boiling temperature ethyl vinyl ether appeared to be preferable. 
We found that the exothermic effect of addition of ethyl vinyl ether to N-H bond in syntheses of larger scale is quite significant. In higher temperatures reaction tends to accelerate and can get out of control. The control reaction can be achieved by portionwise addition of ethyl vinyl ether to the reaction mixture at $28-33{ }^{\circ} \mathrm{C}$ in dichloromethane, with catalytic amount of trifluoroacetic acid (TFA).

The monitoring of reaction of 3,4-diiodo- $1 \mathrm{H}$-pyrazole (6) and 4-bromo-3-iodo- $1 \mathrm{H}$-pyrazole (7) with ethyl vinyl ether indicated the migration of EtOEt protecting group in acidic conditions. After keeping reaction mixture at room temperature for $2 \mathrm{~h}$, in both cases the mixtures of isomers were detected. According GC-MS analysis, the ratios 6a to 6a' and 7a to 7a' were 1:1 (Scheme 2, table 1). After keeping the reaction mixtures at room temperature for prolonged period of time $(20-78$ hours) only traces of 5-iodo isomers 6a' and 7a' were left. 4-Bromo(iodo)-1-(1-ethoxyethyl)-3iodo-1 $H$-pyrazoles 6a and 7a were isolated in excellent yields. Di-substituted EtOEt-protected pyrazole derivatives 8a - 14a were synthesized applying the same method in good yields (Scheme2, Table 1).

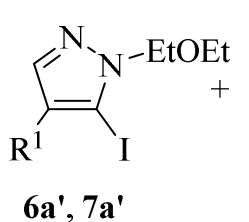

r.t. $2 \mathrm{~h}: 50 \%$

r.t. $20 \mathrm{~h}: 20 \%$

r.t. $78 \mathrm{~h}: 3 \%$

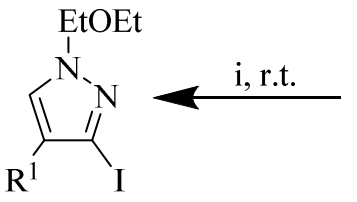

6a, $7 \mathbf{a}$

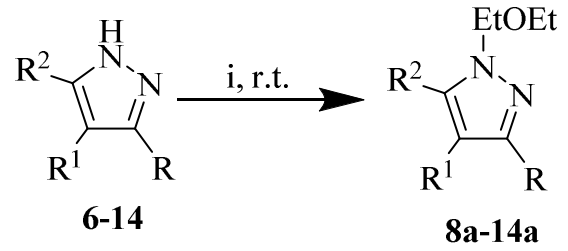

6: $\mathrm{R}^{1}=\mathrm{I} ; 7: \mathrm{R}^{1}=\mathrm{Br}$;

8: $\mathrm{R}=\mathrm{I}, \mathrm{R}^{1}=\mathrm{H}, \mathrm{R}^{2}=\mathrm{CF}_{3} ; \mathbf{9}: \mathrm{R}=\mathrm{I}, \mathrm{R}^{1}=\mathrm{NO}_{2} ; \mathrm{R}^{2}=\mathrm{H}$;

10: $\mathrm{R}=\mathrm{NO}_{2}, \mathrm{R}^{1}=\mathrm{I}, \mathrm{R}^{2}=\mathrm{H} ; \mathbf{1 1}: \mathrm{R}=\mathrm{I}, \mathrm{R}^{1}=\mathrm{COOEt}, \mathrm{R}^{2}=\mathrm{H}$;

12: $\mathrm{R}=\mathrm{I}, \mathrm{R}^{1}=\mathrm{Me}, \mathrm{R}^{2}=\mathrm{H} ; \mathbf{1 3}: \mathrm{R}=\mathrm{Br}, \mathrm{R}^{1}=\mathrm{NO}_{2}, \mathrm{R}^{2}=\mathrm{H}$;

14: $\mathrm{R}=\mathrm{Br}, \mathrm{R}^{1}=\mathrm{COOEt}, \mathrm{R}^{2}=\mathrm{H}$;

Scheme 2. Protection of N-H group in disubstituted $1 H$-pyrazole derivatives 6-14. i: ethyl vinyl ether, TFA, $\mathrm{CH}_{2} \mathrm{Cl}_{2}, 28-33{ }^{\circ} \mathrm{C}$, then room temperature.

It is known that 1-(1-ethoxyethyl)-4-iodo- $1 H$-pyrazole (3a) can be easily converted to Grignard reagents using alkyl magnesium bromides. ${ }^{17} 1$-(1-Ethoxyethyl)- $1 H$-pyrazole-4-carbaldehyde (16) was synthesized in good yield using this intermediate. Thus, we wanted to compare the reactivity of 1-(1-ethoxyethyl)-3-iodo-1H-pyrazole (2a), 4-iodopyrazole 3a and 3,4-diiodopyrazole 6a towards alkyl magnesium halides and to evaluate the possibilities of application of Grignard reagents in derivatization of iodopyrazoles to pyrazole aldehydes. It is known that quantity of final pyrazole aldehydes reflects with the quantity of metalated pyrazoles, because usually these reactions occurs instantaneously. Unfortunately, 3-iodo derivative 2a didn't react with ethylmagnesium bromide. Only $25 \%$ conversion of 2a to aldehyde $\mathbf{1 5}$ was achieved using 2-propylmagnesium bromide lithium bromide (Scheme 3, table 2). Thus, we were expecting that EtMgBr could selectively react with iodo atom in 4-th position of 3,4-diiodo derivative 6a. Full conversion of $\mathbf{6 a}$ was achieved 
within 30 minutes at $+5{ }^{\circ} \mathrm{C}$, however the formation of both available isomers - 1-(1-ethoxyethyl)-4iodo- $1 H$-pyrazole-3-carbaldehyde (17) and 1-(1-ethoxyethyl)-3-iodo- $1 H$-pyrazole-4-carbaldehyde (18) in ratio 1:1 was observed. Decreasing reaction temperature increases selectivity of substitution (at $-10{ }^{\circ} \mathrm{C}$ ratio of $\mathbf{1 7}$ to $\mathbf{1 8}$ is 1 to 2 , at $-40{ }^{\circ} \mathrm{C}$ ratio reaches 1 to 4 ). However the starting compound is not fully consumed, by performing iodo- $\mathrm{EtMgBr}$ exchange reaction at $-10{ }^{\circ} \mathrm{C}$ degrees after 2 hours $-10 \%$ of $\mathbf{6 a}$ was left unreacted, at $-40{ }^{\circ} \mathrm{C}-30 \%$. Analogous bromopyrazoles $4 \mathbf{a}$ and $\mathbf{5 a}$ did not react with alkyl magnesium halides.

Table 1. Synthesis of N-ethoxyethyl protected pyrazoles $\mathbf{6 a - 1 4 a}$

\begin{tabular}{|c|c|c|c|c|c|c|}
\hline \multicolumn{4}{|c|}{ Starting material } & \multirow{2}{*}{ Time, $\mathrm{h}$} & \multirow{2}{*}{ Product Nr. } & \multirow{2}{*}{ Yield, \%* } \\
\hline $\mathrm{Nr}$ & $\mathrm{R}$ & $\mathrm{R}^{1}$ & $\mathrm{R}^{2}$ & & & \\
\hline 6 & I & $\mathrm{I}$ & $\mathrm{H}$ & 78 & $6 \mathbf{a}$ & 86 \\
\hline 7 & I & $\mathrm{Br}$ & $\mathrm{H}$ & 78 & $7 a$ & 77 \\
\hline 8 & I & $\mathrm{H}$ & $\mathrm{CF}_{3}$ & 14 & $\mathbf{8 a}$ & 75 \\
\hline 9 & I & $\mathrm{NO}_{2}$ & $\mathrm{H}$ & 14 & $\mathbf{9 a}$ & 77 \\
\hline 10 & $\mathrm{NO}_{2}$ & I & $\mathrm{H}$ & 14 & $10 \mathbf{a}$ & 80 \\
\hline 11 & I & COOEt & $\mathrm{H}$ & 14 & $11 a$ & 43 \\
\hline 12 & $\mathrm{I}$ & $\mathrm{Me}$ & $\mathrm{H}$ & 78 & $12 \mathbf{a}$ & 80 \\
\hline 13 & $\mathrm{Br}$ & $\mathrm{NO}_{2}$ & $\mathrm{H}$ & 24 & $13 \mathbf{a}$ & 75 \\
\hline 14 & $\mathrm{Br}$ & COOEt & $\mathrm{H}$ & 24 & $14 a$ & 83 \\
\hline
\end{tabular}

*Isolated yield.

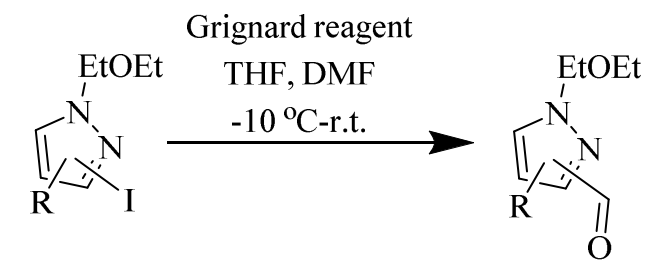

2a, 3a, 6a

$15-18$

Scheme 3. Reaction of 1-(1-ethoxyethyl)-3-iodo-, 4-iodo- and 3,4-diiodo-1H-pyrazole derivatives 2a, 3a, 6a with Grignard reagents.

There are some suggestions for the synthesis of pyrazole derivatives in high yields via halogenlithium exchange reaction. ${ }^{21,22}$ Usually such reactions are performed by direct lithiation at 5-th position or by halogen-lithium exchange reaction of halogen atom at 4-th or 5-th positions of the pyrazole ring. Our experiment has shown, that N-EtOEt group acts as ortho-directing group. Thus, direct ortho lithiation reaction of N-EtOEt protected pyrazole derivatives was performed using nBuLi (1a) or lithium diisopropylamide (LDA) (2a, 3a, 5a) as lithiating agents and pyrazole aldehydes $\mathbf{1 c}-\mathbf{3 c}, \mathbf{5 c}$ were synthesized in good yields (Scheme 4). 
Table 2. Synthesis of N-EtOEt pyrazole aldehydes 15, 16, 17 and 18

\begin{tabular}{|c|c|c|c|c|c|c|}
\hline $\begin{array}{l}\text { Starting } \\
\text { material }\end{array}$ & $\mathrm{R}$ & I & $\begin{array}{l}\text { Grignard } \\
\text { reagents }\end{array}$ & Product & Substituents & $\begin{array}{c}\text { Conversion* } \\
(\text { Yield } * *)\end{array}$ \\
\hline $2 \mathbf{a}$ & $4-\mathrm{H}$ & $3-\mathrm{I}$ & $\begin{array}{l}\mathrm{EtMgBr} \\
\text { 2-PrMgBr/LiBr }\end{array}$ & 15 & 4-H; 3-CHO & $\begin{array}{c}0 \% \\
25 \%(19 \%)\end{array}$ \\
\hline $\mathbf{3 a}$ & $3-\mathrm{H}$ & 4-I & $\mathrm{EtMgBr}$ & 16 & $3-\mathrm{H} ; 4-\mathrm{CHO}$ & $85 \%(72 \%)$ \\
\hline $6 \mathbf{a}$ & $3-\mathrm{I}$ & $4-\mathrm{I}$ & $\mathrm{EtMgBr}$ & $\begin{array}{l}17 \\
18\end{array}$ & $\begin{array}{l}4-\mathrm{I} ; 3-\mathrm{CHO} \\
3-\mathrm{I} ; 4-\mathrm{CHO}\end{array}$ & $\begin{array}{l}30 \%(10 \%) \\
65 \%(55 \%)\end{array}$ \\
\hline
\end{tabular}

* GC-MS data;**Isolated yield.

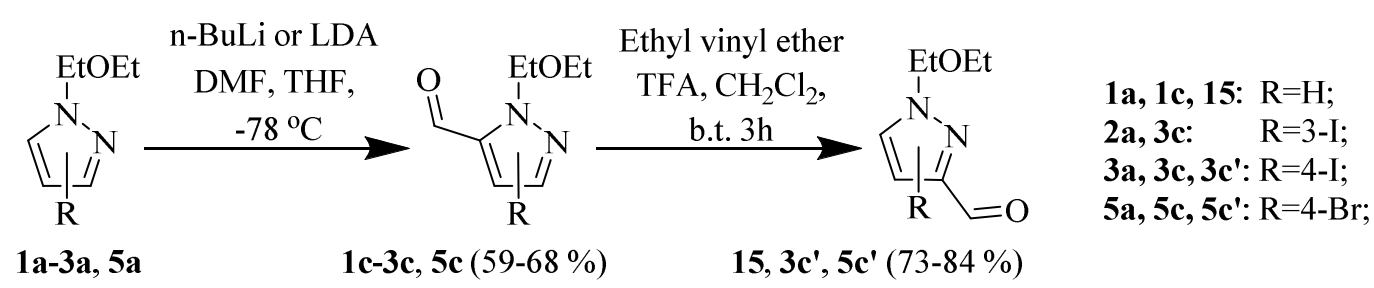

Scheme 4. Reactions of 1-(1-ethoxyethyl)-1H-pyrazole derivatives 1a-3a, 5a with lithium organic compounds

Ethoxyethyl protecting group in compounds $\mathbf{1 c}-\mathbf{3 c}, \mathbf{5 c}$ appeared to be very sensitive to acidic conditions. The migration of protecting group can be performed by heating 1-(1-ethoxyethyl)- $1 H$ pyrazole-5-carbaldehyde (1c), 1-(1-ethoxyethyl)-4-iodo-1 $H$-pyrazole-5-carbaldehyde (3c) or 4bromo-1-(1-ethoxyethyl)-1 $\mathrm{H}$-pyrazole-5-carbaldehyde $(\mathbf{5 c})$ in dichloromethane at $40{ }^{\circ} \mathrm{C}$ for $3 \mathrm{~h}$, using catalytic amount of trifluoroacetic acid and 5\% of ethyl vinyl ether for prevention of deprotection. Thus, 1-(1-ethoxyethyl)-1H-pyrazole-3-carbaldehyde (15), 1-(1-ethoxyethyl)-4-iodo$1 H$-pyrazole-3-carbaldehyde (3c') and 4-bromo-1-(1-ethoxyethyl)-1H-pyrazole-3-carbaldehyde (5c') were synthesized in good yields. Structure of final products was proven by ${ }^{1} \mathrm{H}-\mathrm{NMR}$, but significantly differs only chemical shift of protecting group NCH proton. Also we have noticed that the acidity of silica gel, which was used for purification, was enough for the migration of protecting group via elimination/addition of ethyl vinyl ether. However, migration could be prevented by washing silica gel column with 5\% triethylamine (TEA) solution in dichloromethane before chromatography. Thus, ethoxyethyl protecting group can be recommended as switchable protecting group for synthesis of pyrazole derivatives as well as tetrahydropyran group. ${ }^{20}$

Reactions of substituted 1-(1-protected)-3-iodo- $1 H$-pyrazole derivatives $2 \mathbf{a}-\mathbf{c}, 7 \mathbf{a}, \mathbf{8 a}, 12 \mathbf{a}, 18$ and 19 with phenyl acetylene were successfully performed under standard Sonogashira crosscoupling conditions (Scheme 5). Compounds 20-27 were synthesized in high yields (Table 3). 


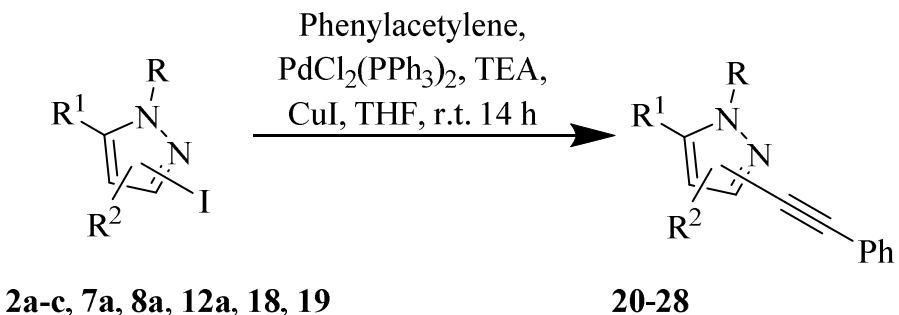

Scheme 5. Sonogashira cross-coupling reaction of substituted iodopyrazole derivatives.

Table 3. Synthesis of compounds 20-30 via Sonogashira cross-coupling reaction.

\begin{tabular}{|c|c|c|c|c|c|c|}
\hline \multicolumn{5}{|c|}{ Starting material } & \multirow{2}{*}{$\begin{array}{c}\text { Product } \\
\text { Nr. }\end{array}$} & \multirow{2}{*}{$\begin{array}{c}\text { Yield, } \\
\% *\end{array}$} \\
\hline $\mathrm{Nr}$ & $\mathrm{R}$ & $\mathrm{R}^{1}$ & $\mathrm{R}^{2}$ & I & & \\
\hline $2 \mathbf{b}$ & Boc & $\mathrm{H}$ & $4-\mathrm{H}$ & $3-\mathrm{I}$ & 20 & 65 \\
\hline $2 a$ & EtOEt & $\mathrm{H}$ & $4-\mathrm{H}$ & 3-I & 21 & 63 \\
\hline $2 c$ & EtOEt & $\mathrm{CHO}$ & $4-\mathrm{H}$ & $3-\mathrm{I}$ & 22 & 80 \\
\hline $7 \mathbf{a}$ & EtOEt & $\mathrm{H}$ & $4-\mathrm{Br}$ & $3-\mathrm{I}$ & 23 & 64 \\
\hline $8 \mathbf{a}$ & EtOEt & $\mathrm{CF}_{3}$ & $4-\mathrm{H}$ & $3-\mathrm{I}$ & 24 & 73 \\
\hline $12 a$ & EtOEt & $\mathrm{H}$ & 4-Me & $3-\mathrm{I}$ & 25 & 70 \\
\hline 18 & EtOEt & $\mathrm{H}$ & 4-CHO & $3-\mathrm{I}$ & 26 & 87 \\
\hline 19 & $\mathrm{Me}$ & $\mathrm{H}$ & 4-H & $3-\mathrm{I}$ & 27 & 82 \\
\hline 9a & EtOEt & $\mathrm{H}$ & $4-\mathrm{NO}_{2}$ & $3-\mathrm{I}$ & - & 0 \\
\hline $11 a$ & EtOEt & $\mathrm{H}$ & 4-COOEt & $3-\mathrm{I}$ & - & 0 \\
\hline $10 a$ & EtOEt & $\mathrm{H}$ & 4-I & $3-\mathrm{NO}_{2}$ & 28 & 58 \\
\hline
\end{tabular}

*Isolated yield

Analogous bromopyrazoles 4a and 5a didn't react with phenyl acetylene under various Sonogashira cross-coupling conditions. Unfortunately 3-iodo-pyrazole derivatives 9a with nitro group and 11a with ethoxycarbonyl substituents at 4-th-pyrazole ring position were also unreactive under various conditions, only starting compounds 9a and 11a were obtained during GC-MS analysis. To the contrary to compound 9a, it's isomer 1-(1-ethoxyethyl)-4-iodo-3-nitro-1Hpyrazole (10a) has formed cross-coupling product $\mathbf{2 8}$ in $58 \%$ yield.

\section{Experimental Section}

General. Melting points were determined in open capillaries and are uncorrected. ${ }^{1} \mathrm{H}$ and ${ }^{13} \mathrm{C}$ NMR spectra were recorded at 400 and $100 \mathrm{MHz}$ respectively on a Brucker 400 instrument with $\mathrm{CDCl}_{3}$ or DMSO- $d_{6}$ solvents using residual solvent peaks as internal standards. Mass spectra were recorded on a Shimadzu QP2010 instrument equipped Restec Rtx-1701 w/Integra-Guard column 
(30 m, $0.25 \mathrm{~mm}$ ID, $0.25 \mu \mathrm{m}$ thickness). HRMS spectra were obtained on a mass spectrometer Dual-ESI Q-TOF 6520 (Agilent Technologies). Silica gel 60 F254 aluminum plates (Merck) were used for TLC analysis. Column chromatography was performed on silica gel $60(0.04-0.063 \mathrm{~mm})$ (Roth).

General synthetic procedure for the synthesis of $\mathrm{N}$-Boc protected pyrazole derivatives $1 \mathrm{~b}-5 \mathrm{~b}$. To a solution of pyrazole 1-5 (1 equiv.) and triethylamine (1.5 equiv.) in dichloromethane (for 0.05 mol of pyrazole $-50 \mathrm{~mL}$ of dichloromethane were user) Di-tert-butyl dicarbonate (1.2 equiv) were added at room temperature and left to stir overnight. Dichloromethane was washed with saturated $\mathrm{NaHCO}_{3}$ solution $\left(1 \times 25 \mathrm{~mL}-\right.$ for $50 \mathrm{~mL}$ of dichloromethane) then with deionized $\mathrm{H}_{2} \mathrm{O}(1 \times 25$ $\mathrm{mL}$ ). Organic layer was dried with anhydrous $\mathrm{Na}_{2} \mathrm{SO}_{4}$, and evaporated under reduced pressure.

tert-Butyl 1H-pyrazole-1-carboxylate (1b). Reaction was performed in $0.069 \mathrm{~mol}$ scale of $1 \mathrm{H}$ pyrazole (1). Purification by distillation gave the titled compound as slightly yellow oil, yield 59\%, $6.8 \mathrm{~g}$, bp $48{ }^{\circ} \mathrm{C}$ at $1.5 \mathrm{mbar}\left(\mathrm{bp} 130{ }^{\circ} \mathrm{C}\right.$ at $2 \mathrm{mmHg}$ ) ${ }^{23}{ }^{1} \mathrm{H}$ NMR (400 MHz, DMSO- $d_{6}$ ) $\delta: 1.58$ (s, $\left.9 \mathrm{H}, 3 \times \mathrm{CH}_{3}\right), 6.53(\mathrm{dd}, J 2.8,1.6 \mathrm{~Hz}, 1 \mathrm{H}, \mathrm{Ar}-\mathrm{H}), 7.80(\mathrm{~d}, J 0.9 \mathrm{~Hz}, 1 \mathrm{H}, \mathrm{Ar}-\mathrm{H}), 8.26(\mathrm{~d}, J 3.6 \mathrm{~Hz}, 1 \mathrm{H}$, Ar-H). ${ }^{13} \mathrm{C}$ NMR (100 MHz, DMSO- $\left.d_{6}\right) \delta: 27.9,85.2,109.5,131.7,144.4,147.6 . \mathrm{MS}, \mathrm{m} / z(\%) 168$ (4), 109 (19), 95 (7), 57 (100).

tert-Butyl 3-iodo-1H-pyrazole-1-carboxylate (2b). Reaction was performed in $0.052 \mathrm{~mol}$ scale of 3 -iodo-1H-pyrazole (2). Purification by recrystallization from $\mathrm{n}$-hexane gave the titled compound as white crystals, yield $78.5 \%, 12 \mathrm{~g}, \mathrm{mp} 82-84^{\circ} \mathrm{C} .{ }^{1} \mathrm{H}$ NMR $\left(400 \mathrm{MHz}, \mathrm{DMSO}-d_{6}\right) \delta: 1.57(\mathrm{~s}, 9 \mathrm{H}$, $\left.3 \times \mathrm{CH}_{3}\right), 6.76(\mathrm{~d}, J 2.8 \mathrm{~Hz}, 1 \mathrm{H}, \mathrm{Ar}-\mathrm{H}), 8.16(\mathrm{~d}, J 2.8 \mathrm{~Hz}, 1 \mathrm{H}, \mathrm{Ar}-\mathrm{H}) .{ }^{13} \mathrm{C}$ NMR $(100 \mathrm{MHz}, \mathrm{DMSO}-$ $\left.d_{6}\right) \delta: 27.9,86.1,104.6,118.2,133.6,146.4 . \mathrm{MS}, \mathrm{m} / z$ (\%) 194 (M-Boc, 100), 167 (25), 128 (2), 40 (36). HRMS (ES) calculated for $[\mathrm{M}+\mathrm{Na}]^{+} \mathrm{C}_{8} \mathrm{H}_{11} \mathrm{IN}_{2} \mathrm{NaO}_{2}$ : 316.9757; found 316.9757.

tert-Butyl 4-iodo- $\mathbf{H}$-pyrazole-1-carboxylate (3b). Reaction was performed in $0.052 \mathrm{~mol}$ scale of 4-iodo-1H-pyrazole (3). Purification by recrystallization from $\mathrm{n}$-hexane gave the titled compound as white crystals, yield 78.5\%, $12 \mathrm{~g}, \mathrm{mp} 72-74{ }^{\circ} \mathrm{C} .{ }^{1} \mathrm{H}$ NMR (400 MHz, DMSO- $\left.d_{6}\right) \delta: 1.57(\mathrm{~s}, 9 \mathrm{H}$, $\left.3 \times \mathrm{CH}_{3}\right), 7.89(\mathrm{~d}, J 0.6 \mathrm{~Hz}, 1 \mathrm{H}, \mathrm{Ar}-\mathrm{H}), 8.46(\mathrm{~d}, J 0.6 \mathrm{~Hz}, 1 \mathrm{H}, \mathrm{Ar}-\mathrm{H}) .{ }^{13} \mathrm{C}$ NMR $(100 \mathrm{MHz}, \mathrm{DMSO}-$ $\left.d_{6}\right) \delta: 27.9,63.8,86.0,135.9,146.4,148.8 . \mathrm{MS}, \mathrm{m} / z$ (\%) 194 (M-Boc, 100), 167 (2), 128 (2), 67 (17), 40 (24). HRMS (ES) calculated for [M+Na] ${ }^{+} \mathrm{C}_{8} \mathrm{H}_{11} \mathrm{IN}_{2} \mathrm{NaO}_{2}: 316.9757$; found 316.9757.

tert-Butyl 3-bromo-1H-pyrazole-1-carboxylate (4b). Reaction was performed in 0.136 mol scale of 3-bromo-1H-pyrazole (4). Purification by recrystallization from n-hexane gave the titled compound as white crystals, yield $80.4 \%, 27 \mathrm{~g}, \mathrm{mp} 33-35{ }^{\circ} \mathrm{C} .{ }^{1} \mathrm{H}$ NMR $\left(400 \mathrm{MHz}, \mathrm{DMSO}-d_{6}\right) \delta$ $1.58\left(\mathrm{~s}, 9 \mathrm{H}, 3 \times \mathrm{CH}_{3}\right), 6.72(\mathrm{~d}, J 2.8 \mathrm{~Hz}, 1 \mathrm{H}, \mathrm{Ar}-\mathrm{H}), 8.30(\mathrm{t}, J 2.8 \mathrm{~Hz}, 1 \mathrm{H}, \mathrm{Ar}-\mathrm{H}) .{ }^{13} \mathrm{C}$ NMR $(100$ MHz, DMSO- $\left.d_{6}\right) \delta 27.8,86.3,112.6,131.8,134.1,146.4$. MS, $m / z(\%) 148: 146$ (M-Boc : M-Boc, 100 : 100), 121 (21), 119 (21), 67 (9), 40 (51). HRMS (ES) calculated for $[\mathrm{M}+\mathrm{Na}]^{+}$ $\mathrm{C}_{8} \mathrm{H}_{11} \mathrm{BrN}_{2} \mathrm{NaO}_{2}$ : 268.9896; found 268.9896 .

tert-Butyl 4-bromo-1H-pyrazole-1-carboxylate (5b). Reaction was performed in 0.068 mol scale of 4-bromo-1 $\mathrm{H}$-pyrazole (5). Purification by recrystallization from $\mathrm{n}$-hexane gave the titled compound as white crystals, yield 79.6\%, $13.4 \mathrm{~g}, \mathrm{mp} .43-45{ }^{\circ} \mathrm{C} .{ }^{1} \mathrm{H}$ NMR $\left(400 \mathrm{MHz}\right.$, DMSO- $\left.d_{6}\right) \delta$ $1.57\left(\mathrm{~s}, 9 \mathrm{H}, 3 \times \mathrm{CH}_{3}\right), 7.94$ (d, J $\left.0.6 \mathrm{~Hz}, 1 \mathrm{H}, \mathrm{Ar}-\mathrm{H}\right), 8.54$ (d, $\left.J 0.6 \mathrm{~Hz}, 1 \mathrm{H}, \mathrm{Ar}-\mathrm{H}\right) .{ }^{13} \mathrm{C} \mathrm{NMR}(100$ 
MHz, DMSO- $\left.d_{6}\right) \delta 27.8,86.1,97.2,131.7,144.7,146.5$. MS, $m / z(\%) 148: 146$ (M-Boc : M-Boc, 100 : 100), 121 (21), 119 (21), 94 (13), 92 (13), 67 (12), 40 (33). HRMS (ES) calculated for $[\mathrm{M}+\mathrm{Na}]^{+} \mathrm{C}_{8} \mathrm{H}_{11} \mathrm{BrN}_{2} \mathrm{NaO}_{2}$ : 268.9896; found 268.9896 .

General synthetic procedure for the synthesis of N-EtOEt protected pyrazole derivatives 1a13a.

To a solution of pyrazole 1-13 (1 equiv.) and trifluoroacetic acid (0.01 equiv.) in dichloromethane (for $1 \mathrm{~mol}$ of pyrazole - $1 \mathrm{~L}$ of dichloromethane were used) ethyl vinyl ether (1.27 equiv.) was added dropwise, keeping the temperature between $28-32{ }^{\circ} \mathrm{C}$ (exothermic reaction) and left to stir at room temperature for 12-78 hours. Dichloromethane was washed with saturated $\mathrm{NaHCO}_{3}$ solution $\left(1 \times 250 \mathrm{~mL}-\right.$ for $1 \mathrm{~L}$ of dichloromethane) then with deionized $\mathrm{H}_{2} \mathrm{O}(1 \times 250 \mathrm{~mL})$. Organic layer was dried with anhydrous $\mathrm{Na}_{2} \mathrm{SO}_{4}$, and evaporated under reduced pressure. Products were purified by distillation or recrystallization.

1-(1-Ethoxyethyl)-1H-pyrazole (1a). Reaction was performed in $20.48 \mathrm{~mol}$ scale of $1 \mathrm{H}$-pyrazole (1). Purification by distillation gave the titled compound as slightly yellow oil, yield 93\%, $2686 \mathrm{~g}$, bp $52{ }^{\circ} \mathrm{C}$ at 9 mbar. (bp $71-74^{\circ} \mathrm{C}$ at $20 \mathrm{mmHg}$ ). ${ }^{24}{ }^{1} \mathrm{H}$ NMR (400 MHz, DMSO- $\left.d_{6}\right) \delta: 1.02$ (t, $J 7.0$ $\left.\mathrm{Hz}, 3 \mathrm{H}, \mathrm{CH}_{2} \mathrm{CH}_{3}\right), 1.58$ (d, J 6.0 Hz, 3H, $\mathrm{CHCH}_{3}$ ), 3.14 (dq, J 9.6, 7.1 Hz, 1H, $\mathrm{CHHCH}_{3}$ ), 3.44 $3.36\left(\mathrm{~m}, 1 \mathrm{H}, \mathrm{CHHCH}_{3}\right), 5.55(\mathrm{q}, J 6.0 \mathrm{~Hz}, 1 \mathrm{H}, \mathrm{NCH}), 7.89$ (t, $\left.J 2.0 \mathrm{~Hz}, 1 \mathrm{H}, \mathrm{Ar}-\mathrm{H}\right), 7.49$ (d, $J 1.3$ $\mathrm{Hz}, 1 \mathrm{H}, \mathrm{Ar}-\mathrm{H}), 7.89$ (d, $J 2.0 \mathrm{~Hz}, 1 \mathrm{H}, \mathrm{Ar}-\mathrm{H}) .{ }^{13} \mathrm{C}$ NMR (100 MHz, DMSO- $\left.d_{6}\right) \delta: 15.2,21.7,63.3$, 86.4, 106.2, 128.3, 139.1. MS, $\mathrm{m} / z$ (\%) 140 (1), 125 (2), 96 (49), 73 (12), 45 (100).

3-Iodo-1-(1-ethoxyethyl)-1H-pyrazole (2a). Reaction was performed in $0.26 \mathrm{~mol}$ scale of 3-jodo$1 \mathrm{H}$-pyrazole (2). Purification by distillation gave the titled compound as slightly yellow oil, yield 92\%, $70 \mathrm{~g}$, bp $72{ }^{\circ} \mathrm{C}(1.8 \mathrm{mbar}) .{ }^{1} \mathrm{H}$ NMR $\left(400 \mathrm{MHz}, \mathrm{DMSO}-d_{6}\right) \delta: 1.03$ (t, $\left.J 7.0 \mathrm{~Hz}, 3 \mathrm{H}, \mathrm{CH}_{2} \mathrm{CH}_{3}\right)$, $1.56\left(\mathrm{~d}, J 6.0 \mathrm{~Hz}, 3 \mathrm{H}, \mathrm{CHCH}_{3}\right), 3.17\left(\mathrm{tt}, J 9.6,5.4 \mathrm{~Hz}, 1 \mathrm{H}, \mathrm{CHHCH}_{3}\right), 3.45-3.36(\mathrm{~m}, 1 \mathrm{H}$, $\left.\mathrm{CHHCH}_{3}\right), 5.54(\mathrm{q}, J 6.0 \mathrm{~Hz}, 1 \mathrm{H}, \mathrm{NCH}), 6.53(\mathrm{~d}, J 2.4 \mathrm{~Hz}, 1 \mathrm{H}, \mathrm{Ar}-\mathrm{H}), 7.84(\mathrm{~d}, J 2.4 \mathrm{~Hz}, 1 \mathrm{H}, \mathrm{Ar}-$ H). ${ }^{13} \mathrm{C}$ NMR (100 MHz, DMSO- $\left.d_{6}\right) \delta: 15.2,21.6,63.6,86.9,96.3,114.9,131.2 . \mathrm{MS}, \mathrm{m} / z(\%) 266$ (7), 222 (41), 207 (14), 193 (11), 95 (26), 73 (56), 45 (100). HRMS (ES) calculated for [M+Na] $]^{+}$ $\mathrm{C}_{7} \mathrm{H}_{11} \mathrm{IN}_{2} \mathrm{NaO}$ : 288.9808; found 288.9809 .

4-Iodo-1-(1-ethoxyethyl)-1H-pyrazole (3a). Reaction was performed in $9.43 \mathrm{~mol}$ scale of 4-iodo$1 \mathrm{H}$-pyrazole (3). Purification by distillation gave the titled compound as white oil, yield 93\%, 2327 g, bp $70{ }^{\circ} \mathrm{C}$ (2.2 mbar). Lit. ${ }^{19}$ bp $74-76^{\circ} \mathrm{C}\left(0.5\right.$ torr). ${ }^{1} \mathrm{H}$ NMR $\left(400 \mathrm{MHz}\right.$, DMSO- $\left.d_{6}\right) \delta: 1.03$ (t, $J 7.0$ $\mathrm{Hz}, 3 \mathrm{H}, \mathrm{CH}_{2} \mathrm{CH}_{3}$ ), 1.57 (d, J $6.0 \mathrm{~Hz}, 3 \mathrm{H}, \mathrm{CHCH}_{3}$ ), 3.17 (dq, J 9.6, 7.0 Hz, 1H, $\mathrm{CHHCH}_{3}$ ), 3.40 (dq, $J$ 9.6, 7.0 Hz, 1H, $\left.\mathrm{CHHCH}_{3}\right), 5.54$ (q, J $\left.6.0 \mathrm{~Hz}, 1 \mathrm{H}, \mathrm{NCH}\right), 7.58$ (s, 1H, Ar-H), $8.13(\mathrm{~s}, 1 \mathrm{H}, \mathrm{Ar}-\mathrm{H})$. ${ }^{13} \mathrm{C}$ NMR $\left(100 \mathrm{MHz}, \mathrm{DMSO}-d_{6}\right) \delta: 15.1,21.5,58.5,63.6,87.0,133.0,144.0 . \mathrm{MS}, \mathrm{m} / \mathrm{z}(\%) 266$ (31), 222 (42), 194 (48), 72 (91), 45 (100). HRMS (ES) calculated for $\left[\mathrm{M}+\mathrm{Na}^{+} \mathrm{C}_{7} \mathrm{H}_{11} \mathrm{IN}_{2} \mathrm{NaO}\right.$ : 288.9808; found 288.9809 .

3-Bromo-1-(1-ethoxyethyl)-1H-pyrazole (4a). Reaction was performed in $0.136 \mathrm{~mol}$ scale of 3bromo-1H-pyrazole (4). Purification by distillation gave the titled compound as white oil, yield 85.6\%, $25.5 \mathrm{~g}$, bp $68{ }^{\circ} \mathrm{C}$ at 3.6 mbar. ${ }^{1} \mathrm{H}$ NMR (400 MHz, DMSO- $\left.d_{6}\right) \delta: 1.04(\mathrm{t}, J 7.0 \mathrm{~Hz}, 3 \mathrm{H}$, $\left.\mathrm{CH}_{2} \mathrm{CH}_{3}\right), 1.57\left(\mathrm{~d}, J 6.0 \mathrm{~Hz}, 3 \mathrm{H}, \mathrm{CHCH}_{3}\right), 3.19$ (dq, $J$ 9.6, 7.0 Hz, $\left.1 \mathrm{H}, \mathrm{CHHCH}_{3}\right), 3.38-3.46(\mathrm{~m}$, 
$\left.1 \mathrm{H}, \mathrm{CHHCH}_{3}\right), 5.52(\mathrm{q}, J 6.0 \mathrm{~Hz}, 1 \mathrm{H}, \mathrm{NCH}), 6.45(\mathrm{~d}, J 2.4 \mathrm{~Hz}, 1 \mathrm{H}, \mathrm{Ar}-\mathrm{H}), 7.95(\mathrm{~d}, J 2.4 \mathrm{~Hz}, 1 \mathrm{H}$, Ar-H). ${ }^{13} \mathrm{C}$ NMR (100 MHz, DMSO- $\left.d_{6}\right) \delta 15.1,21.5,63.6,87.1,108.8,125.3,131.6 . \mathrm{MS}, \mathrm{m} / z(\%)$ 220 : 218 (2 : 2), 176 (16), 174 (16), 161 (10), 159 (10), 148 (9), 146 (9), 94 (12), 73 (96), 45 (100). HRMS (ES) calculated for $[\mathrm{M}+\mathrm{Na}]^{+} \mathrm{C}_{7} \mathrm{H}_{11} \mathrm{BrN}_{2} \mathrm{NaO}$ : 240.9947; found 240.9946 .

4-Bromo-1-(1-ethoxyethyl)-1H-pyrazole (5a). Reaction was performed in $0.136 \mathrm{~mol}$ scale of 3bromo-1H-pyrazole (5). Purification by distillation gave the titled compound as white oil, yield $73.3 \%, 21.8 \mathrm{~g}$, bp $55{ }^{\circ} \mathrm{C}$ at 2.6 mbar. ${ }^{1} \mathrm{H}$ NMR (400 MHz, DMSO- $\left.d_{6}\right) \delta: 1.03(\mathrm{t}, J 7.0 \mathrm{~Hz}, 3 \mathrm{H}$, $\left.\mathrm{CH}_{2} \mathrm{CH}_{3}\right), 1.57\left(\mathrm{~d}, J 6.0 \mathrm{~Hz}, 3 \mathrm{H}, \mathrm{CHCH}_{3}\right), 3.19$ (dq, $J$ 9.6, 7.0 Hz, 1H, $\left.\mathrm{CHHCH}_{3}\right), 3.41$ (dq, $J$ 9.6, $\left.7.0 \mathrm{~Hz}, 1 \mathrm{H}, \mathrm{CHHCH}_{3}\right), 5.52(\mathrm{q}, J 6.0 \mathrm{~Hz}, 1 \mathrm{H}, \mathrm{NCH}), 7.60(\mathrm{~s}, 1 \mathrm{H}, \mathrm{Ar}-\mathrm{H}), 8.18(\mathrm{~s}, 1 \mathrm{H}, \mathrm{Ar}-\mathrm{H}) .{ }^{13} \mathrm{C}$ NMR (101 MHz, DMSO- $\left.d_{6}\right) \delta 15.1,21.5,63.6,87.4,93.1,128.8,139.5 . \mathrm{MS}, \mathrm{m} / z(\%) 220: 218(5$ : 5), 176 (16), 174 (16), 148 (12), 146 (12), 94 (13), 73 (74), 45 (100). HRMS (ES) calculated for $[\mathrm{M}+\mathrm{Na}]^{+} \mathrm{C}_{7} \mathrm{H}_{11} \mathrm{BrN}_{2} \mathrm{NaO}$ : 240.9947; found 240.9946 .

1-(1-Ethoxyethyl)-3,4-diiodo-1H-pyrazole (6a). Reaction was performed in $0.047 \mathrm{~mol}$ scale of 3,4-diiodo-1H-pyrazole (6). Purification by recrystallization from $\mathrm{n}$-hexane gave the titled compound as slightly yellow crystals, yield $85.9 \%, 15.8 \mathrm{~g}, \mathrm{mp} 55-57{ }^{\circ} \mathrm{C} .{ }^{1} \mathrm{H}$ NMR $(400 \mathrm{MHz}$, DMSO-d $\left.d_{6}\right) \delta: 1.04\left(\mathrm{t}, J 7.0 \mathrm{~Hz}, 3 \mathrm{H}, \mathrm{CH}_{2} \mathrm{CH}_{3}\right), 1.56\left(\mathrm{~d}, J 6.0 \mathrm{~Hz}, 3 \mathrm{H}, \mathrm{CHCH}_{3}\right), 3.20$ (dq, J 9.6, 7.0 $\left.\mathrm{Hz}, 1 \mathrm{H}, \mathrm{CHHCH}_{3}\right), 3.41$ (dq, J 9.6, 7.0 Hz, 1H, $\left.\mathrm{CHHCH}_{3}\right), 5.53$ (q, J 6.0 Hz, 1H, NCH), 8.08 (s, $1 \mathrm{H}, \mathrm{Ar}-\mathrm{H}) .{ }^{13} \mathrm{C}$ NMR $\left(100 \mathrm{MHz}, \mathrm{DMSO}-d_{6}\right) \delta: 15.1,21.5,63.9,73.1,87.5,108.5,135.2 . \mathrm{MS}, \mathrm{m} / \mathrm{z}$ (\%) 392 (33), 320 (61), 221 (21), 72 (74), 45 (100). HRMS (ES) calculated for [M+Na $]^{+}$ $\mathrm{C}_{7} \mathrm{H}_{10} \mathrm{I}_{2} \mathrm{~N}_{2} \mathrm{NaO}: 414.8775$; found 414.8768 .

4-Bromo-1-(1-ethoxyethyl)-3-iodo-1H-pyrazole (7a). Reaction was performed in $0.037 \mathrm{~mol}$ scale of 4-bromo-3-iodo- $1 \mathrm{H}$-pyrazole (7). Purification by recrystallization from $\mathrm{n}$-hexane gave the titled compound as slightly yellow crystals, yield 78.6\%, $10.03 \mathrm{~g}, \mathrm{mp} \mathrm{59-60}{ }^{\circ} \mathrm{C} .{ }^{1} \mathrm{H}$ NMR $(400 \mathrm{MHz}$, DMSO- $\left.d_{6}\right) \delta: 1.04\left(\mathrm{t}, J 7.0 \mathrm{~Hz}, 3 \mathrm{H}, \mathrm{CH}_{2} \mathrm{CH}_{3}\right), 1.56\left(\mathrm{~d}, J 6.0 \mathrm{~Hz}, 3 \mathrm{H}, \mathrm{CHCH}_{3}\right), 3.21$ (dq, $J$ 9.6, 7.0 $\left.\mathrm{Hz}, 1 \mathrm{H}, \mathrm{CHHCH}_{3}\right), 3.42\left(\mathrm{dq}, J\right.$ 9.6, 7.0 Hz, 1H, $\left.\mathrm{CHHCH}_{3}\right), 5.52(\mathrm{q}, J 6.0 \mathrm{~Hz}, 1 \mathrm{H}, \mathrm{NCH}), 8.19$ (s, $1 \mathrm{H}, \mathrm{Ar}-\mathrm{H}) .{ }^{13} \mathrm{C}$ NMR $\left(100 \mathrm{MHz}, \mathrm{DMSO}-d_{6}\right) \delta: 15.1,21.4,63.9,87.9,102.6,102.7,130.5 . \mathrm{MS}, \mathrm{m} / \mathrm{z}$ (\%) $346: 344$ (8:8), 302 (11), 300 (11), 175 (10), 73 (92), 45 (100). HRMS (ES) calculated for $[\mathrm{M}+\mathrm{Na}]^{+} \mathrm{C}_{7} \mathrm{H}_{10} \mathrm{BrIN}{ }_{2} \mathrm{NaO}$ : 366.8913; found 366.8916 .

1-(1-Ethoxyethyl)-3-iodo-5-(trifluoromethyl)-1H-pyrazole (8a). Reaction was performed in 0.02 mol scale of 3-iodo-5-(trifluoromethyl)-1 $H$-pyrazole (8). Purification by column chromatography (ethyl acetate in n-hexane) gave the titled compound as slightly yellow oil, yield $74.9 \%, 5 \mathrm{~g} .{ }^{1} \mathrm{H}$ NMR (400 MHz, DMSO- $d_{6}$ ) $\delta: 1.07$ (t, $J 7.0 \mathrm{~Hz}, 3 \mathrm{H}, \mathrm{CH}_{2} \mathrm{CH}_{3}$ ), 1.62 (d, J $5.9 \mathrm{~Hz}, 3 \mathrm{H}, \mathrm{CHCH}_{3}$ ), $3.20\left(\mathrm{dq}, J\right.$ 9.4, 7.0 Hz, 1H, $\left.\mathrm{CHHCH}_{3}\right), 3.46\left(\mathrm{dq}, J\right.$ 9.3, 7.0 Hz, 1H, $\left.\mathrm{CHHCH}_{3}\right), 5.81(\mathrm{q}, J 5.9 \mathrm{~Hz}$, $1 \mathrm{H}, \mathrm{NCH}), 7.08(\mathrm{~s}, 1 \mathrm{H}, \mathrm{Ar}-\mathrm{H}) .{ }^{13} \mathrm{C}$ NMR $\left(100 \mathrm{MHz}, \mathrm{DMSO}-d_{6}\right) \delta: 15.1,21.4,26.8,63.7,87.1$, 87.2, 114.2 (2 signals), 120.1, 122.7, 143.3, 143.7. MS, m/z (\%) 334 (0.4), 289 (7), 262 (6), 163 (18), 73 (100), 45 (74). HRMS (ES) calculated for [M+Na] $]^{+} \mathrm{C}_{8} \mathrm{H}_{10} \mathrm{~F}_{3} \mathrm{IN}_{2} \mathrm{NaO}$ : 356.9682; found 356.9685 .

1-(1-Ethoxyethyl)-3-iodo-4-nitro-1H-pyrazole (9a). Reaction was performed in $0.021 \mathrm{~mol}$ scale of 3-iodo-4-nitro-1H-pyrazole (9). Purification by recrystallization from n-hexane : ethyl acetate mixture $(10: 1)$ gave titled compound as slightly yellow crystals, yield $77 \%, 5.01 \mathrm{~g}, \mathrm{mp} 82-84{ }^{\circ} \mathrm{C}$. 
${ }^{1} \mathrm{H}$ NMR (400 MHz, $\left.\mathrm{CDCl}_{3}\right) \delta: 1.23\left(\mathrm{t}, J 7.1 \mathrm{~Hz}, 3 \mathrm{H}, \mathrm{CH}_{2} \mathrm{CH}_{3}\right), 1.69\left(\mathrm{~d}, J 6.0 \mathrm{~Hz}, 3 \mathrm{H}, \mathrm{CHCH}_{3}\right)$, $3.62-3.45\left(\mathrm{~m}, 2 \mathrm{H}, \mathrm{CH}_{2} \mathrm{CH}_{3}\right), 5.54(\mathrm{q}, J 6.0 \mathrm{~Hz}, 1 \mathrm{H}, \mathrm{NCH}), 8.33(\mathrm{~s}, 1 \mathrm{H}, \mathrm{Ar}-\mathrm{H}) .{ }^{13} \mathrm{C}$ NMR $(100$ $\left.\mathrm{MHz}, \mathrm{CDCl}_{3}\right) \delta: 14.8,22.4,65.5,90.1,92.5,127.5,139.4 . \mathrm{MS}, \mathrm{m} / z$ (\%) 311 (2), 266 (4), 223 (25), 73 (100), 45 (92). HRMS (ES) calculated for $[\mathrm{M}+\mathrm{Na}]^{+} \mathrm{C}_{7} \mathrm{H}_{10} \mathrm{IN}_{3} \mathrm{NaO}_{3}: 333.9659$; found 333.9658.

1-(1-Ethoxyethyl)-4-iodo-3-nitro-1H-pyrazole (10a). Reaction was performed in 0.042 mol scale starting of 4-iodo-3-nitro-1H-pyrazole (10). Purification by recrystallization from n-hexane : ethyl acetate $(10: 1)$ mixture gave titled compound as slightly yellow crystals, yield $80 \%, 10.4 \mathrm{~g}, \mathrm{mp} 84-$ $85{ }^{\circ} \mathrm{C} .{ }^{1} \mathrm{H}$ NMR $\left(400 \mathrm{MHz}, \mathrm{DMSO}-d_{6}\right) \delta: 1.07\left(\mathrm{t}, J 7.0 \mathrm{~Hz}, 3 \mathrm{H}, \mathrm{CH}_{2} \mathrm{CH}_{3}\right), 1.61(\mathrm{~d}, J 5.9 \mathrm{~Hz}, 3 \mathrm{H}$, $\left.\mathrm{CHCH}_{3}\right), 3.28\left(\mathrm{dq}, J\right.$ 9.5, 7.0 Hz, 1H, $\left.\mathrm{CHHCH}_{3}\right), 3.49\left(\mathrm{dq}, J\right.$ 9.5, 7.0 Hz, 1H, $\left.\mathrm{CHHCH}_{3}\right), 5.66(\mathrm{q}, J$ $5.9 \mathrm{~Hz}, 1 \mathrm{H}, \mathrm{NCH}), 8.49$ (s, $1 \mathrm{H}, \mathrm{Ar}-\mathrm{H}) .{ }^{13} \mathrm{C} \mathrm{NMR}\left(100 \mathrm{MHz}, \mathrm{DMSO}-d_{6}\right) \delta: 15.1,21.4,57.32,64.5$, 89.2, 138.4, 155.1. MS, $m / z$ (\%) 311 (9), 267 (11), 223 (2), 73 (100), 45 (80). HRMS (ES) calculated for $[\mathrm{M}+\mathrm{Na}]^{+} \mathrm{C}_{7} \mathrm{H}_{10} \mathrm{IN}_{3} \mathrm{NaO}_{3}: 333.9659$; found 333.9654 .

Ethyl 1-(1-ethoxyethyl)-3-iodo-1H-pyrazole-4-carboxylate (11a). Reaction was performed in $0.0188 \mathrm{~mol}$ scale starting from ethyl 3-iodo- $1 \mathrm{H}$-pyrazole-4-carboxylate (11). Purification by recrystallization from $n$-hexane : ethyl acetate mixture $(10: 0.5)$ gave titled compound as slightly yellow crystals, yield $43 \%, 2.67 \mathrm{~g}, \mathrm{mp} 65-67{ }^{\circ} \mathrm{C} .{ }^{1} \mathrm{H} \mathrm{NMR}\left(400 \mathrm{MHz}, \mathrm{CDCl}_{3}\right) \delta: 1.20(\mathrm{t}, J 7.0 \mathrm{~Hz}$, $\left.3 \mathrm{H}, \mathrm{CH}_{2} \mathrm{CH}_{3}\right), 1.40\left(\mathrm{t}, J 7.1 \mathrm{~Hz}, 3 \mathrm{H}, \mathrm{COOCH}_{2} \mathrm{CH}_{3}\right), 1.67$ (d, J $\left.6.0 \mathrm{~Hz}, 3 \mathrm{H}, \mathrm{CHCH}_{3}\right), 3.57-3.37$ $\left(\mathrm{m}, 2 \mathrm{H}, \mathrm{OCH}_{2} \mathrm{CH}_{3}\right), 4.38\left(\mathrm{q}, J 7.1 \mathrm{~Hz}, 2 \mathrm{H}, \mathrm{COOCH}_{2} \mathrm{CH}_{3}\right), 5.53(\mathrm{q}, J 6.0 \mathrm{~Hz}, 1 \mathrm{H}, \mathrm{NCH}), 8.02$ (s, $1 \mathrm{H}, \mathrm{Ar}-\mathrm{H}) .{ }^{13} \mathrm{C}$ NMR $\left(100 \mathrm{MHz}, \mathrm{CDCl}_{3}\right) \delta: 14.3,14.8,22.3,60.5,64.9,88.7,97.5,118.4,131.1$, 161.7. MS, m/z (\%) 338 (6), 294 (49), 221 (25), 167 (50), 73 (99), 45 (100). HRMS (ES) calculated for $[\mathrm{M}+\mathrm{Na}]^{+} \mathrm{C}_{10} \mathrm{H}_{15} \mathrm{IN}_{2} \mathrm{NaO}_{3}: 361.0019$; found 361.0018 .

1-(1-Ethoxyethyl)-3-iodo-4-methyl-1H-pyrazole (12a). Reaction was performed in $0.0192 \mathrm{~mol}$ scale starting from ethyl 3-iodo-4-methyl-1H-pyrazole (12). Purification by column chromatography (ethyl acetate in n-hexane, gradient mode) gave titled compound as yellow oil, yield 80\%, 4.3 g. ${ }^{1} \mathrm{H}$ NMR (400 MHz, $\mathrm{CDCl}_{3}$ ) $\delta: 1.03$ (t, $\left.J 7.0 \mathrm{~Hz}, 3 \mathrm{H}, \mathrm{CH}_{2} \mathrm{CH}_{3}\right), 1.54$ (d, J $6.0 \mathrm{~Hz}$, $3 \mathrm{H}, \mathrm{CHCH}_{3}$ ), 1.92 (d, J $\left.0.7 \mathrm{~Hz}, 3 \mathrm{H}, \mathrm{CH}_{3}\right), 3.17$ (dq, J 9.5, 7.1 Hz, 1H, $\mathrm{CHHCH}_{3}$ ), 3.39 (dq, J 9.5, $\left.7.0 \mathrm{~Hz}, 1 \mathrm{H}, \mathrm{CHHCH}_{3}\right), 5.45$ (q, J $\left.6.0 \mathrm{~Hz}, 1 \mathrm{H}, \mathrm{NCH}\right), 7.69$ (d, J 0.7 Hz, 1H, Ar-H). ${ }^{13} \mathrm{C} \mathrm{NMR}(101$ MHz, DMSO- $\left.d_{6}\right) \delta 11.2,15.2,21.6,63.5,86.9,101.8,121.1,127.9 . \mathrm{MS}, \mathrm{m} / z$ (\%) 280 (13), 236 (34), 221 (15), 208 (39), 109 (36), 93 (11), 73 (49), 45 (100). HRMS (ES) calculated for [M+Na] ${ }^{+}$ $\mathrm{C}_{8} \mathrm{H}_{13} \mathrm{IN}_{2} \mathrm{NaO}$ : 302.9965; found 302.9964 .

3-Bromo-1-(1-ethoxyethyl)-4-nitro-1H-pyrazole (13a). Reaction was performed in $0.026 \mathrm{~mol}$ scale starting from ethyl 3-bromo-4-nitro-1H-pyrazole (13). Purification by recrystallization from n-hexane : toluene $(20: 1)$ mixture gave titled compound as white crystals, yield $75 \%, 5.2 \mathrm{~g}$, mp 59-60 ${ }^{\circ} \mathrm{C} .{ }^{1} \mathrm{H}$ NMR $(400 \mathrm{MHz}, \mathrm{DMSO}-d 6) \delta: 1.08\left(\mathrm{t}, J 7.0 \mathrm{~Hz}, 3 \mathrm{H}, \mathrm{CH}_{2} \mathrm{CH}_{3}\right), 1.61(\mathrm{~d}, J 5.9 \mathrm{~Hz}, 3 \mathrm{H}$, $\left.\mathrm{CHCH}_{3}\right), 3.37-3.27\left(\mathrm{~m}, 1 \mathrm{H}, \mathrm{CHHCH}_{3}\right), 3.49$ (dq, J 9.5, 7.0 Hz, 1H, $\left.\mathrm{CHHCH}_{3}\right), 5.62$ (q, J 5.9 Hz, $1 \mathrm{H}, \mathrm{NCH}), 9.18(\mathrm{~s}, 1 \mathrm{H}, \mathrm{Ar}-\mathrm{H}) .{ }^{13} \mathrm{C}$ NMR (100 MHz, DMSO- $\left.d_{6}\right) \delta 15.0,21.1,64.4,88.9,122.3$, 132.7, 133.1. MS, m/z (\%) $266: 264$ (0.2:0.2), 220 (6), 218 (6), 177 (10), 175 (10), 73 (100), 45 (95). HRMS (ES) calculated for $[\mathrm{M}+\mathrm{Na}]^{+} \mathrm{C}_{7} \mathrm{H}_{10} \mathrm{BrN}_{3} \mathrm{NaO}_{3}: 285.9798$; found 285.9797.

Ethyl 3-bromo-1-(1-ethoxyethyl)-1H-pyrazole-4-carboxylate (14a). Reaction was performed in $0.023 \mathrm{~mol}$ scale starting from ethyl 3-bromo-4-nitro-1H-pyrazole (13). Purification by 
recrystallization from n-hexane: toluene (20:1) mixture gave titled compound as white crystals, yield $82.8 \%, 5.5 \mathrm{~g}, \mathrm{mp} 66-67{ }^{\circ} \mathrm{C} .{ }^{1} \mathrm{H}$ NMR $\left(400 \mathrm{MHz}, \mathrm{CDCl}_{3}\right) \delta: 1.05$ (t, $\left.J 7.0 \mathrm{~Hz}, 3 \mathrm{H}, \mathrm{CH}_{2} \mathrm{CH}_{3}\right)$, 1.28 (t, J 7.1 Hz, 3H, $\left.\mathrm{COOCH}_{2} \mathrm{CH}_{3}\right), 1.58$ (d, J $\left.5.9 \mathrm{~Hz}, 3 \mathrm{H}, \mathrm{CHCH}_{3}\right), 3.23$ (dq, J 9.5, 7.0 Hz, $1 \mathrm{H}$, $\left.\mathrm{OCHHCH}_{3}\right), 3.45$ (dq, $J$ 9.5, 7.0 Hz, $\left.1 \mathrm{H}, \mathrm{OCHHCH}_{3}\right), 4.23$ (q, $\left.J 7.1 \mathrm{~Hz}, 2 \mathrm{H}, \mathrm{COOCH}_{2} \mathrm{CH}_{3}\right), 5.59$ (q, J $5.9 \mathrm{~Hz}, 1 \mathrm{H}, \mathrm{NCH}), 8.57$ (s, 1H, Ar-H). ${ }^{13} \mathrm{C}$ NMR (101 MHz, DMSO-d6) $\delta 14.6,15.1,21.3$, 60.5, 64.0, 87.8, 113.1, 127.3, 135.5, 161.3. MS, m/z (\%) $292: 290$ (0.4 : 0.4), 248 (16), 246 (16), 233 (12), 231 (12), 167 (25), 73 (99), 45 (100). HRMS (ES) calculated for $[\mathrm{M}+\mathrm{Na}]^{+}$ $\mathrm{C}_{10} \mathrm{H}_{15} \mathrm{BrN}_{2} \mathrm{NaO}_{3}$ : 313.0158; found 313.0156.

General synthetic procedure for the reaction of pyrazole compounds $2 \mathrm{a}$, 3a and $6 \mathrm{a}$ with Grignard reagents.

To a suspension of magnesium (1.3 equiv) in THF (for $1 \mathrm{~mol}$ of magnesium $700 \mathrm{~mL}$ of THF were used), at the boiling temperature under argon atmosphere, ethyl bromide (1.4 equiv) was added dropwise and left to stir at same temperature for one hour. Then the reaction mixture was cooled down to given temperature and solution of starting pyrazole derivative $\mathbf{2 a}, \mathbf{3 a}$ or $\mathbf{6 a}$ (1 equiv) in THF (for $1 \mathrm{~mol}$ of pyrazole $350 \mathrm{~mL}$ of THF were used) was added dropwise at same temperature. After 1 hour of stirring at the same temperature dimethylformamide (1.5 equiv) was added to the reaction mixture dropwise and left to warm to room temperature overnight. Then saturated $\mathrm{NH}_{4} \mathrm{Cl}$ (5 equiv) solution in deionized water were added to the reaction mixture, organic layer was separated and $\mathrm{NH}_{4} \mathrm{Cl}$ solution was extracted with dichloromethane (for $1 \mathrm{~mol}$ of pyrazole $0.5 \mathrm{~L}$ of dichloromethane were used) twice. Organic layers were combined, washed with deionized water (for $1 \mathrm{~mol}$ of pyrazole $0.5 \mathrm{~L}$ of water were used), dried with anhydrous $\mathrm{Na}_{2} \mathrm{SO}_{4}$ and evaporated under reduced pressure. Products were purified by distillation or column chromatography.

1-(1-Ethoxyethyl)-1H-pyrazole-3-carbaldehyde (15). Reaction was performed in $0.00188 \mathrm{~mol}$ scale of 1-(1-ethoxyethyl)-3-iodo- $1 H$-pyrazole (2a), iodo - i- $\mathrm{PrMgBr} \mathrm{LiBr}$ exchange reaction was performed at $0-+5{ }^{\circ} \mathrm{C}$. Purification by column chromatography (ethyl acetate in n-hexane, gradient mode) gave titled product as white oil, yield 19\%, $0.06 \mathrm{~g} .{ }^{1} \mathrm{H} \mathrm{NMR}\left(400 \mathrm{MHz}, \mathrm{CDCl}_{3}\right) \delta$ : $1.16\left(\mathrm{t}, J 7.0 \mathrm{~Hz}, 3 \mathrm{H}, \mathrm{CH}_{2} \mathrm{CH}_{3}\right), 1.69\left(\mathrm{~d}, J 6.0 \mathrm{~Hz}, 3 \mathrm{H}, \mathrm{CHCH}_{3}\right), 3.34(\mathrm{dq}, J 9.4,7.0 \mathrm{~Hz}, 1 \mathrm{H}$, $\left.\mathrm{CHHCH}_{3}\right), 3.50\left(\mathrm{dq}, J\right.$ 9.4, $\left.7.0 \mathrm{~Hz}, 1 \mathrm{H}, \mathrm{CHHCH}_{3}\right), 5.59$ (q, J $\left.6.0 \mathrm{~Hz}, 1 \mathrm{H}, \mathrm{NCH}\right), 6.87$ (d, J $2.5 \mathrm{~Hz}$, 1H,Ar-CH), 7.65 (dd, J 2.6, $0.7 \mathrm{~Hz}, 1 \mathrm{H}, \mathrm{Ar}-\mathrm{H}), 9.98$ (d, $J 0.7 \mathrm{~Hz}, 1 \mathrm{H}, \mathrm{CHO}) .{ }^{1} \mathrm{H}$ NMR $(400 \mathrm{MHz}$, DMSO- $\left.d_{6}\right) \delta: 1.05$ (t, $\left.J 7.0 \mathrm{~Hz}, 3 \mathrm{H}, \mathrm{CH} 2 \mathrm{CH} 3\right), 1.64$ (d, $\left.J 6.0 \mathrm{~Hz}, 3 \mathrm{H}, \mathrm{CHCH} 3\right), 3.22$ (dq, $J$ 9.6, 7.0 $\mathrm{Hz}, 1 \mathrm{H}, \mathrm{CHHCH} 3), 3.52-3.42$ (m, 1H, CHHCH3), 5.70 (q, J $6.0 \mathrm{~Hz}, 1 \mathrm{H}, \mathrm{NCH}), 6.84$ (d, J $2.5 \mathrm{~Hz}$, $1 \mathrm{H}, \mathrm{Ar}-\mathrm{H}), 8.12(\mathrm{dd}, J$ 2.5, $0.6 \mathrm{~Hz}, 1 \mathrm{H}, \mathrm{Ar}-\mathrm{H}), 9.92(\mathrm{~d}, J 0.6 \mathrm{~Hz}, 1 \mathrm{H}, \mathrm{CHO}) .{ }^{13} \mathrm{C}$ NMR $(100 \mathrm{MHz}$, DMSO-d6) $\delta: 15.2,21.7,64.0,87.9,106.1,131.2,151.1,187.3 . \mathrm{MS}, \mathrm{m} / z$ (\%) 168 (2), 153 (3), 139 (30), 124 (83) 73 (99), 45 (100). HRMS (ES) calculated for $[\mathrm{M}+\mathrm{Na}]^{+} \mathrm{C}_{8} \mathrm{H}_{12} \mathrm{~N}_{2} \mathrm{NaO}_{2}$ : 191.0791; found 191.0790 .

1-(1-Ethoxyethyl)-1H-pyrazole-4-carbaldehyde (16). Reaction was performed in $8.27 \mathrm{~mol}$ scale of 1-(1-ethoxyethyl)-4-iodo- $1 H$-pyrazole (3a), iodo - EtMgBr exchange reaction was performed at $0-+2{ }^{\circ} \mathrm{C}$. Purification by distillation gave titled product as white oil, yield $72 \%, 1000 \mathrm{~g}$, bp $87^{\circ} \mathrm{C}$ at 2 mbar. ${ }^{1} \mathrm{H}$ NMR $\left(400 \mathrm{MHz}, \mathrm{CDCl}_{3}\right) \delta: 1.17\left(\mathrm{t}, J 7.0 \mathrm{~Hz}, 3 \mathrm{H}, \mathrm{CH}_{2} \mathrm{CH}_{3}\right), 1.68(\mathrm{~d}, J 6.0 \mathrm{~Hz}, 3 \mathrm{H}$, $\left.\mathrm{CHCH}_{3}\right), 3.38\left(\mathrm{dq}, J\right.$ 9.4, $\left.7.0 \mathrm{~Hz}, 1 \mathrm{H}, \mathrm{CHHCH}_{3}\right), 3.50\left(\mathrm{dq}, J\right.$ 9.4, $\left.7.0 \mathrm{~Hz}, 1 \mathrm{H}, \mathrm{CHHCH}_{3}\right), 5.55$ (q, $J$ 
$6.0 \mathrm{~Hz}, 1 \mathrm{H}, \mathrm{NCH}), 7.98$ (d, J 0.5 Hz, 1H, Ar-H), 8.15 (d, J $0.5 \mathrm{~Hz}, 1 \mathrm{H}, \mathrm{Ar}-\mathrm{H}), 9.90$ (s, 1H, CHO). ${ }^{1} \mathrm{H}$ NMR $\left(400 \mathrm{MHz}, \mathrm{DMSO}-d_{6}\right) \delta: 1.05\left(\mathrm{t}, J 7.0 \mathrm{~Hz}, 3 \mathrm{H}, \mathrm{CH}_{2} \mathrm{CH}_{3}\right), 1.61\left(\mathrm{~d}, J 6.0 \mathrm{~Hz}, 3 \mathrm{H}, \mathrm{CHCH}_{3}\right)$, $3.23\left(\mathrm{dq}, J\right.$ 9.6, 7.0 Hz, 1H, $\left.\mathrm{CHHCH}_{3}\right), 3.46\left(\mathrm{dq}, J\right.$ 9.6, 7.0 Hz, 1H, $\left.\mathrm{CHHCH}_{3}\right), 5.64$ (q, J $6.0 \mathrm{~Hz}$, $1 \mathrm{H}, \mathrm{NCH}), 8.04(\mathrm{~s}, 1 \mathrm{H}, \mathrm{Ar}-\mathrm{H}), 8.66(\mathrm{~s}, 1 \mathrm{H}, \mathrm{Ar}-\mathrm{H}), 9.84(\mathrm{~s}, 1 \mathrm{H}, \mathrm{CHO}) .{ }^{13} \mathrm{C}$ NMR $(100 \mathrm{MHz}$, DMSO-d6) $\delta: 15.1,21.6,63.9,87.4,124.5,133.8,140.0,185.4$. MS, $m / z$ (\%) 168 (1), 153 (1), 124 (59), 95 (22), 73 (60), 45 (100). HRMS (ES) calculated for $[\mathrm{M}+\mathrm{Na}]^{+} \mathrm{C}_{8} \mathrm{H}_{12} \mathrm{~N}_{2} \mathrm{NaO}_{2}$ : 191.0791; found 191.0793 .

1-(1-Ethoxyethyl)-3-iodo-1 $H$-pyrazole-4-carbaldehyde (18) and 1-(1-ethoxyethyl)-4-iodo-1 $H$ pyrazole-3-carbaldehyde (17). Reaction was performed in $0.01 \mathrm{~mol}$ scale of 1-(1-ethoxyethyl)3,4-diiodo- $1 H$-pyrazole (6a) iodo - EtMgBr exchange reaction was performed at $-10--8{ }^{\circ} \mathrm{C}$. Purification by column chromatography (ethyl acetate in $n$-hexane $(1: 4))$ gave product $\mathbf{1 8}(\mathrm{Rf}=$ 0.4 ) as slightly yellow oil, yield 55\%, $1.65 \mathrm{~g} .{ }^{1} \mathrm{H}$ NMR (400 MHz, DMSO- $\left.d_{6}\right) \delta: 1.06(\mathrm{t}, J 7.0 \mathrm{~Hz}$, $\left.3 \mathrm{H}, \mathrm{CH}_{2} \mathrm{CH}_{3}\right), 1.60\left(\mathrm{~d}, J 6.0 \mathrm{~Hz}, 3 \mathrm{H}, \mathrm{CHCH}_{3}\right), 3.25\left(\mathrm{dq}, J 9.6,7.0 \mathrm{~Hz}, 1 \mathrm{H}, \mathrm{CHHCH}_{3}\right), 3.47$ (dq, $J$ 9.6, 7.0 Hz, 1H, $\left.\mathrm{CHHCH}_{3}\right), 5.64(\mathrm{q}, J 6.0 \mathrm{~Hz}, 1 \mathrm{H}, \mathrm{NCH}), 8.60(\mathrm{~s}, 1 \mathrm{H}, \mathrm{Ar}-\mathrm{H}), 9.69(\mathrm{~s}, 1 \mathrm{H}$, CHO).${ }^{13} \mathrm{C}$ NMR (100 MHz, DMSO- $\left.d_{6}\right) \delta: 15.1,21.5,64.1,87.9,100.4,124.6,135.4,185.5$. MS, $\mathrm{m} / z$ (\%) 294 (5), 250 (62), 221 (11), 73 (100), 45 (96). HRMS (ES) calculated for [M+Na] ${ }^{+}$ $\mathrm{C}_{8} \mathrm{H}_{11} \mathrm{IN}_{2} \mathrm{NaO}_{2}: \quad 316.9757$; found 316.9760 . And 1-(1-ethoxyethyl)-4-iodo-1H-pyrazole-3carbaldehyde $(\mathbf{1 7})(\mathrm{Rf}=0.5)$ as slightly yellow oil, yield 10\%, $0.3 \mathrm{~g} .{ }^{1} \mathrm{H}$ NMR (400 MHz, DMSO$\left.d_{6}\right) \delta: 1.06\left(\mathrm{t}, J 7.0 \mathrm{~Hz}, 3 \mathrm{H}, \mathrm{CH}_{2} \mathrm{CH}_{3}\right), 1.63\left(\mathrm{~d}, J 6.0 \mathrm{~Hz}, 3 \mathrm{H}, \mathrm{CHCH}_{3}\right), 3.24(\mathrm{dq}, J 9.6,7.0 \mathrm{~Hz}, 1 \mathrm{H}$, $\left.\mathrm{CHHCH}_{3}\right), 3.47\left(\mathrm{dq}, J\right.$ 9.5, 7.0 Hz, $\left.1 \mathrm{H}, \mathrm{CHHCH}_{3}\right), 5.68(\mathrm{q}, J 6.0 \mathrm{~Hz}, 1 \mathrm{H}, \mathrm{NCH}), 8.37$ (d, $J 0.6 \mathrm{~Hz}$, $1 \mathrm{H}, \mathrm{Ar}-\mathrm{H}), 9.88(\mathrm{~d}, J 0.6 \mathrm{~Hz}, 1 \mathrm{H}, \mathrm{CHO}) .{ }^{13} \mathrm{C} \mathrm{NMR}\left(100 \mathrm{MHz}, \mathrm{DMSO}-d_{6}\right) \delta: 15.1,21.5,59.6,64.2$, 88.48, 136.6, 148.3, 186.5. MS, m/z (\%) 294 (23), 265 (21), 250 (14), 221 (7), 73 (89), 45 (100). HRMS (ES) calculated for $[\mathrm{M}+\mathrm{Na}]^{+} \mathrm{C}_{8} \mathrm{H}_{11} \mathrm{IN}_{2} \mathrm{NaO}_{2}: 316.9757$; found 316.9760 .

1-(1-Ethoxyethyl)-1H-pyrazole-5-carbaldehyde (1c). To a solution of protected pyrazole $1 \mathbf{a}$ (644 $\mathrm{g}$, $4.6 \mathrm{~mol})$ in $6 \mathrm{~L}$ of tetrahydrofuran, under argon atmosphere, $2 \mathrm{~L}$ of $\mathrm{n}$-BuLi $(5 \mathrm{~mol}, 2.5 \mathrm{M}$ in $\mathrm{n}$ hexane) were added dropwise at $-78{ }^{\circ} \mathrm{C}$ and left to stir at same temperature for 1 hour. Then dimethylformamide $(5.5 \mathrm{~mol})$ were added to the reaction mixture $\left(\right.$ at $\left.-78^{\circ} \mathrm{C}\right)$ and left to warm to the room temperature, and then additionally stirred at room temperature overnight. Then reaction was cooled down to $5{ }^{\circ} \mathrm{C}$ and $5 \mathrm{~L}$ of saturated $\mathrm{NH}_{4} \mathrm{Cl}$ solution in water was added, organic layer was separated, and concentrated under reduced pressure. Crude product was dissolved in $2.5 \mathrm{~L}$ dichloromethane and extracted with $\mathrm{NH}_{4} \mathrm{Cl}$ solution in water (same $\mathrm{NH}_{4} \mathrm{Cl}$ solution with was used for quenching), water layer was washed with dichloromethane $(2 \times 250 \mathrm{~mL})$. Organic layers were combined and washed with deionized water $(2 \times 250 \mathrm{~mL})$, dried with anhydrous $\mathrm{Na}_{2} \mathrm{SO}_{4}$ and evaporated under reduced pressure. Purification by distillation gave titled compound as slightly yellow oil, yield $65 \%, 500 \mathrm{~g}$, bp $56^{\circ} \mathrm{C}$ at 1.3 mbar. ${ }^{1} \mathrm{H} \mathrm{NMR}\left(400 \mathrm{MHz}, \mathrm{CDCl}^{3}\right) \delta: 1.14(\mathrm{t}, J 7.0 \mathrm{~Hz}$, $\left.3 \mathrm{H}, \mathrm{CH}_{2} \mathrm{CH}_{3}\right), 1.72\left(\mathrm{~d}, J 6.0 \mathrm{~Hz}, 3 \mathrm{H}, \mathrm{CHCH}_{3}\right), 3.29$ (dq, J 9.3, 7.0 Hz, 1H, $\left.\mathrm{CHHCH}_{3}\right), 3.47$ (dq, $J$ 9.3, 7.0 Hz, 1H, $\left.\mathrm{CHHCH}_{3}\right), 6.33$ (q, J $\left.6.0 \mathrm{~Hz}, 1 \mathrm{H}, \mathrm{NCH}\right), 6.94$ (d, J $\left.2.0 \mathrm{~Hz}, 1 \mathrm{H}, \mathrm{Ar}-\mathrm{H}\right), 7.63$ (d, J $1.9 \mathrm{~Hz}, 1 \mathrm{H}, \mathrm{Ar}-\mathrm{H}), 9.99$ (s, Hz, 1H, CHO). ${ }^{1} \mathrm{H}$ NMR (400 MHz, DMSO-d 6 ) $\delta: 1.02$ (t, $J 7.0 \mathrm{~Hz}, 3 \mathrm{H}$, $\mathrm{CH}_{2} \mathrm{CH}_{3}$ ), 1.64 (d, J $5.9 \mathrm{~Hz}, 3 \mathrm{H}, \mathrm{CHCH}_{3}$ ), 3.16 (dq, J 9.4, 7.0 Hz, 1H, $\mathrm{CHHCH}_{3}$ ), 3.41 (dq, J 9.4, $\left.7.0 \mathrm{~Hz}, 1 \mathrm{H}, \mathrm{CHHCH}_{3}\right), 6.30(\mathrm{q}, J 5.9 \mathrm{~Hz}, 1 \mathrm{H}, \mathrm{NCH}), 7.14$ (d, J $\left.1.9 \mathrm{~Hz}, 1 \mathrm{H}, \mathrm{Ar}-\mathrm{H}\right), 7.74$ (d, J 1.9 
$\mathrm{Hz}, 1 \mathrm{H}, \mathrm{Ar}-\mathrm{H}), 10.02$ (s, $1 \mathrm{H}, \mathrm{CHO}) .{ }^{13} \mathrm{C} \mathrm{NMR}\left(100 \mathrm{MHz}, \mathrm{DMSO}-d_{6}\right) \delta: 15.2,21.6,63.3,85.3$, 115.1, 140.0, 140.4, 182.0. MS, m/z (\%) 168 (1), 153 (2), 139 (37), 124 (22), 73 (80), 45 (100). HRMS (ES) calculated for [M+Na] ${ }^{+} \mathrm{C}_{8} \mathrm{H}_{12} \mathrm{~N}_{2} \mathrm{NaO}_{2}$ : 191.0791; found 191.0793.

General synthetic procedure for the reaction of pyrazole compounds $2 \mathrm{a}, 3 \mathrm{a}$ and $5 \mathrm{a}$ with LDA. To dry tetrahydrofurane (for $0.019 \mathrm{~mol}$ of starting material $40 \mathrm{~mL}$ of tetrahydrofurane was used), 2.5 $\mathrm{M}$ n-BuLi solution in $\mathrm{n}$-hexane (1.1 equiv.) was added dropwise under argon atmosphere at -78 ${ }^{\circ} \mathrm{C}$ temperature, following by addition of diisopropylamine (1.2 equiv.) at $-78{ }^{\circ} \mathrm{C}$ degree and left to stir at same temperature for $30 \mathrm{~min}$. Then pyrazole $\mathbf{2 a}$, 3a or $\mathbf{5 a}$ (1 equiv.) solution in tetrahydrofurane (for $0,019 \mathrm{~mol}$ of protected pyrazole $-5 \mathrm{~mL}$ of THF were used) was added to the reaction mixture at $-78{ }^{\circ} \mathrm{C}$ temperature and left to stir at the same temperature for additional $30 \mathrm{~min}$. Then dimethylformamide (1.3 equiv.) solution in tetrahydrofurane (for $0.025 \mathrm{~mol}$ of dimethylformamide $-5 \mathrm{~mL}$ of THF were used) were added dropwise at $-78{ }^{\circ} \mathrm{C}$ degree and left to warm to room temperature overnight. Reaction mixture was cooled down to $5{ }^{\circ} \mathrm{C}$ temperature and quenched with saturated $\mathrm{NH}_{4} \mathrm{Cl}$ solution in deionized water (for 0.019 mol of starting material -12 $\mathrm{mL}$ of saturated $\mathrm{NH}_{4} \mathrm{Cl}$ solution were used). Organic layer was separated, inorganic layer was extracted with dichloromethane $(3 \times 10 \mathrm{~mL})$. Organic layers were combined and washed with deionized water $(2 \times 10 \mathrm{~mL})$, dried with anhydrous $\mathrm{Na}_{2} \mathrm{SO}_{4}$ and evaporated under reduced pressure.

1-(1-Ethoxyethyl)-3-iodo-1H-pyrazole-5-carbaldehyde (2c). Reaction was performed in 0.019 mol scale of 1-(1-ethoxyethyl)-3-iodo- $1 H$-pyrazole (2a). Purification by recrystallization of nhexane gave titled compound as slightly yellow crystals, yield 59\%, $3.29 \mathrm{~g}, \mathrm{mp} \mathrm{70-72}{ }^{\circ} \mathrm{C} .{ }^{1} \mathrm{H} \mathrm{NMR}$ (400 MHz, DMSO-d $)_{6} \delta: 1.03$ (t, J 7.0 Hz, 3H, $\mathrm{CH}_{2} \mathrm{CH}_{3}$ ), 1.62 (d, J $5.9 \mathrm{~Hz}, 3 \mathrm{H}, \mathrm{CHCH}_{3}$ ), 3.20 (dq, $J$ 9.4, 7.0 Hz, 1H, $\left.\mathrm{CHHCH}_{3}\right), 3.43$ (dq, J 9.4, 7.0 Hz, 1H, $\left.\mathrm{CHHCH}_{3}\right), 6.25$ (q, J $5.9 \mathrm{~Hz}, 1 \mathrm{H}, \mathrm{NCH}$ ), 7.32 (s, $1 \mathrm{H}, \mathrm{Ar}-\mathrm{H}), 9.95$ (s, 1H, CHO). ${ }^{13} \mathrm{C}$ NMR (100 MHz, DMSO-d $\left.d_{6}\right) \delta: 15.1,21.6,63.6,85.8$, 97.5, 122.4, 142.2, 181.3. MS, m/z (\%) 294 (5), 265 (7), 250 (25), 235 (5), 123 (11), 73 (100), 45 (96). HRMS (ES) calculated for $[\mathrm{M}+\mathrm{Na}]^{+} \mathrm{C}_{8} \mathrm{H}_{11} \mathrm{IN}_{2} \mathrm{NaO}_{2}$ : 316.9757; found 316.9760 .

1-(1-Ethoxyethyl)-4-iodo-1H-pyrazole-5-carbaldehyde (3c). Reaction was performed in 0.113 mol scale of 1-(1-ethoxyethyl)-4-iodo-1 $H$-pyrazole (3a). Purification by recrystallization from nhexane gave titled compound as slightly yellow crystals, yield $68 \%, 22.6 \mathrm{~g}, \mathrm{mp} 53-54{ }^{\circ} \mathrm{C} .{ }^{1} \mathrm{H}$ NMR $\left(400 \mathrm{MHz}, \mathrm{CDCl}_{3}\right) \delta: 1.17$ (t, $\left.J 7.0 \mathrm{~Hz}, 3 \mathrm{H}, \mathrm{CH}_{2} \mathrm{CH}_{3}\right), 1.71$ (d, J $\left.5.9 \mathrm{~Hz}, 3 \mathrm{H}, \mathrm{CHCH}_{3}\right), 3.33$ (dq, $J$ 9.3, 7.0 Hz, 1H, $\mathrm{CHHCH}_{3}$ ), 3.49 (dq, J 9.3, 7.0 Hz, 1H, CHHCH3), 6.48 (q, $J 5.9 \mathrm{~Hz}, 1 \mathrm{H}, \mathrm{NCH}$ ), $7.72(\mathrm{~d}, J 0.4 \mathrm{~Hz}, 1 \mathrm{H}, \mathrm{Ar}-\mathrm{H}), 9.90$ (s, 1H, CHO). ${ }^{1} \mathrm{H}$ NMR (400 MHz, DMSO- $\left.d_{6}\right) \delta: 1.02$ (t, $J 7.0$ $\mathrm{Hz}, 3 \mathrm{H}, \mathrm{CH}_{2} \mathrm{CH}_{3}$ ), 1.60 (d, J $5.9 \mathrm{~Hz}, 3 \mathrm{H}, \mathrm{CHCH}_{3}$ ), 3.18 (dq, J 9.4, 7.0 Hz, 1H, $\mathrm{CHHCH}_{3}$ ), 3.41 (dq, $J$ 9.4, $\left.7.0 \mathrm{~Hz}, 1 \mathrm{H}, \mathrm{CHHCH}_{3}\right), 6.34(\mathrm{q}, J 5.9 \mathrm{~Hz}, 1 \mathrm{H}, \mathrm{NCH}), 7.88(\mathrm{~s}, 1 \mathrm{H}, \mathrm{Ar}-\mathrm{H}), 9.87(\mathrm{~s}, 1 \mathrm{H}, \mathrm{CHO})$. ${ }^{13} \mathrm{C}$ NMR $\left(100 \mathrm{MHz}, \mathrm{DMSO}-d_{6}\right) \delta: 15.2,21.3,63.5,71.4,85.3,137.3,145.9,182.0 . \mathrm{MS}, \mathrm{m} / z(\%)$ 294 (14), 265 (2), 250 (15), 235 (4), 223 (9), 73 (94), 45 (100). HRMS (ES) calculated for [M+Na] $]^{+}$ $\mathrm{C}_{8} \mathrm{H}_{11} \mathrm{IN}_{2} \mathrm{NaO}_{2}$ : 316.9757; found 316.9760 .

4-Bromo-1-(1-ethoxyethyl)-1H-pyrazole-5-carbaldehyde (5c). Reaction was performed in 0.0137 mol scale of 4-bromo-1-(1-ethoxyethyl)-1H-pyrazole (5a). Purification by column chromatography (ethyl acetate in $\mathrm{n}$-hexane, gradient mode) gave titled compound as slightly yellow oil, yield 73.9\%, 2.5 g. ${ }^{1} \mathrm{H}$ NMR (400 MHz, DMSO-d 6 ) $\delta: 1.03$ (t, $J 7.0 \mathrm{~Hz}, 3 \mathrm{H}, \mathrm{CH}_{2} \mathrm{CH}_{3}$ ), 1.61 (d, 
$\left.J 5.9 \mathrm{~Hz}, 3 \mathrm{H}, \mathrm{CHCH}_{3}\right), 3.22\left(\mathrm{dq}, J 9.5,7.0 \mathrm{~Hz}, 1 \mathrm{H}, \mathrm{CHHCH}_{3}\right), 3.43(\mathrm{dq}, J 9.5,7.0 \mathrm{~Hz}, 1 \mathrm{H}$, $\left.\mathrm{CHHCH}_{3}\right), 6.31(\mathrm{q}, J 5.9 \mathrm{~Hz}, 1 \mathrm{H}, \mathrm{NCH}), 7.92(\mathrm{~s}, 1 \mathrm{H}, \mathrm{Ar}-\mathrm{H}), 9.95(\mathrm{~s}, 1 \mathrm{H}, \mathrm{CHO}) .{ }^{13} \mathrm{C}$ NMR $(100$ MHz, DMSO- $\left.d_{6}\right) \delta: 15.1,21.3,63.6,85.8,102.8,135.6,141.0,180.7$. HRMS (ES) calculated for $[\mathrm{M}+\mathrm{Na}]^{+} \mathrm{C}_{8} \mathrm{H}_{11} \mathrm{BrN}_{2} \mathrm{NaO}_{2}$ : 268.9896; found 268.9899 .

General synthetic procedure for the ethoxyethyl group rearrangement of the compounds 1c and $3 c$.

To a solution of pyrazole $\mathbf{1 c}$ or $\mathbf{3 c}$ ( 1 equiv.) and trifluoroacetic acid (0.01 equiv.) in dichloromethane (for $1 \mathrm{~mol}$ of pyrazole - $1 \mathrm{~L}$ of dichloromethane were used) ethyl vinyl ether $(0.1$ equiv.) was added. Then reaction mixture was heated at $40{ }^{\circ} \mathrm{C}$ degree for $1-2$ hours and cooled down to room temperature. Dichloromethane was washed with saturated $\mathrm{NaHCO}_{3}$ solution $(1 \times$ $250 \mathrm{~mL}$ - for $1 \mathrm{~L}$ of dichloromethane) then with deionized $\mathrm{H}_{2} \mathrm{O}(1 \times 250 \mathrm{~mL})$. Organic layer was dried with anhydrous $\mathrm{Na}_{2} \mathrm{SO}_{4}$, and evaporated under reduced pressure. Products were purified by column chromatography.

1-(1-Ethoxyethyl)-1H-pyrazole-3-carbaldehyde (14). Reaction was performed in $0.012 \mathrm{~mol}$ scale of 1-(1-ethoxyethyl)-1H-pyrazole-5-carbaldehyde (1c). Purification by column chromatography (ethyl acetate in n-hexane) gave titled compound, yield $84.3 \%, 1.7 \mathrm{~g}$.

1-(1-Ethoxyethyl)-4-iodo-1H-pyrazole-3-carbaldehyde (3c'). Reaction was performed in 0.007 mol scale of 1-(1-ethoxyethyl)-4-iodo-1H-pyrazole-5-carbaldehyde (3c). Purification by column chromatography (ethyl acetate in n-hexane) gave titled compound as slightly yellow crystals, yield $72.9 \%, 1.5 \mathrm{~g}$.

General synthetic procedure for the Sonogashira cross-coupling reaction.

To the solution of protected pyrazole 2a-c, 7a, 8a, 12a, 17, 18 or 10a $(1 \mathrm{mmol})$ in $2 \mathrm{~mL}$ of dry THF $\mathrm{PdCl}_{2}\left(\mathrm{PPh}_{3}\right)_{2}(14.04 \mathrm{mg}, 0.02 \mathrm{mmol})$ and triethylamine $(0.25 \mathrm{~g}, 2.5 \mathrm{mmol})$ were added. Reaction mixture was flushed with argon and acetylene $(1.2 \mathrm{mmol})$ were added to the reaction mixture dropwise, following by addition of $\mathrm{CuI}(1.9 \mathrm{mg}, 0.01 \mathrm{mmol})$ and left to stir at room temperature overnight. $5 \mathrm{~mL}$ of deionized water were added to the reaction mixture, organic layer was separated and product was extracted with dichloromethane $(2 \times 5 \mathrm{~mL})$, organic layers were combined and washed with $5 \mathrm{~mL}$ of water, dried with anhydrous $\mathrm{Na}_{2} \mathrm{SO}_{4}$ and evaporated under reduced pressure.

tert-Butyl 3-(phenylethynyl)-1H-pyrazole-1-carboxylate (20). tert-Butyl 3-iodo-1H-pyrazole-1carboxylate $(\mathbf{2 b})$ was used, purification by column chromatography (ethyl acetate : n-hexane $(1$ : 8), $\mathrm{Rf}=0.4$ ) gave titled compound as white crystals, yield $64.9 \%, 0.174 \mathrm{~g}, \mathrm{mp} 89-91{ }^{\circ} \mathrm{C} .{ }^{1} \mathrm{H} \mathrm{NMR}$ $\left(400 \mathrm{MHz}, \mathrm{DMSO}-d_{6}\right) \delta: 1.60\left(\mathrm{~s}, 9 \mathrm{H}, \mathrm{CH}\left(\mathrm{CH}_{3}\right)_{3}\right), 6.80(\mathrm{~d}, J 2.8 \mathrm{~Hz}, 1 \mathrm{H}, \mathrm{CH}) 7.53-7.44(\mathrm{~m}, 3 \mathrm{H}$, $\mathrm{Ph}-\mathrm{CH}), 7.64-7.58$ (m, 2H, Ph-CH), 8.38 (d, $J 2.8 \mathrm{~Hz}, 1 \mathrm{H}, \mathrm{CH}) .{ }^{13} \mathrm{C}$ NMR (100 MHz, DMSO- $\left.d_{6}\right)$ $\delta: 27.9,82.0,86.2,91.5,112.8,121.6,129.4,130.0,132.0,132.8,138.6,146.9 . \mathrm{MS}, \mathrm{m} / z(\%) 168$ (M-Boc, 100), 139 (36), 114 (14), 84 (6). HRMS (ES) calculated for $\left[\mathrm{M}+\mathrm{Na}^{+} \mathrm{C}_{16} \mathrm{H}_{16} \mathrm{~N}_{2} \mathrm{NaO}_{2}\right.$ : 291.1104; found 291.1103.

1-(1-Ethoxyethyl)-3-(phenylethynyl)-1H-pyrazole (21). 1-(1-Ethoxyethyl)-3-iodo-1H-pyrazole (2a) was used, purification by column chromatography (ethyl acetate : n-hexane $(1: 4), \mathrm{Rf}=0.6$ ) gave titled compound as slightly yellow oil, yield 62.5\%, 0.15 g. ${ }^{1} \mathrm{H}$ NMR (400 MHz, DMSO- $\left.d_{6}\right) \delta$ : $1.05\left(\mathrm{t}, J 7.0 \mathrm{~Hz}, 3 \mathrm{H}, \mathrm{CH}_{2} \mathrm{CH}_{3}\right), 1.61\left(\mathrm{~d}, J 6.0 \mathrm{~Hz}, 3 \mathrm{H}, \mathrm{CHCH}_{3}\right), 3.21$ (dq, J 9.6, 7.0 Hz, $1 \mathrm{H}$, 
$\left.\mathrm{CHHCH}_{3}\right), 3.44\left(\mathrm{dq}, J\right.$ 9.6, $\left.7.0 \mathrm{~Hz}, 1 \mathrm{H}, \mathrm{CHHCH}_{3}\right), 5.58\left(\mathrm{q}, J 6.0 \mathrm{~Hz}, 1 \mathrm{H}, \mathrm{CHCH}_{3}\right), 6.61$ (d, J 2.4 $\mathrm{Hz}, 1 \mathrm{H}, \mathrm{CH}) 7.47-7.41$ (m, 3H, Ph-CH), $7.59-7.53$ (m, 2H, Ph-CH), 8.01 (d, J 2.4 Hz, 1H, CH). ${ }^{13} \mathrm{C}$ NMR (100 MHz, DMSO- $\left.d_{6}\right) \delta: 15.2,21.6,63.7,83.6,87.1,89.4,110.3,122.4,129.3,129.4$, 129.7, 131.7, 133.7. MS, m/z (\%) 240 (29), 196 (33), 168 (100), 139 (20), 73 (21), 45 (58). HRMS (ES) calculated for $[\mathrm{M}+\mathrm{Na}]^{+} \mathrm{C}_{15} \mathrm{H}_{16} \mathrm{~N}_{2} \mathrm{NaO}: 263.1155$; found 263.1154 .

1-(1-Ethoxyethyl)-3-(phenylethynyl)-1H-pyrazole-5-carbaldehyde (22). 1-(1-Ethoxyethyl)-3iodo- $1 H$-pyrazole-5-carbaldehyde (2c) was used, purification by column chromatography (ethyl acetate : n-hexane $(1: 4), \mathrm{Rf}=0.5)$ gave titled compound as slightly yellow crystals, yield $80 \%$, 0.215 g, mp 64-65 ${ }^{\circ} \mathrm{C} .{ }^{1} \mathrm{H}$ NMR (400 MHz, DMSO- $\left.d_{6}\right) \delta: 1.05\left(\mathrm{t}, J 7.0 \mathrm{~Hz}, 3 \mathrm{H}, \mathrm{CH}_{2} \mathrm{CH}_{3}\right), 1.66(\mathrm{~d}$, $\left.J 5.9 \mathrm{~Hz}, 3 \mathrm{H}, \mathrm{CHCH}_{3}\right), 3.24\left(\mathrm{dq}, J 9.4,7.0 \mathrm{~Hz}, 1 \mathrm{H}, \mathrm{CHHCH}_{3}\right), 3.46(\mathrm{dq}, J$ 9.4, $7.0 \mathrm{~Hz}, 1 \mathrm{H}$, $\left.\mathrm{CHHCH}_{3}\right), 6.32\left(\mathrm{q}, J 5.9 \mathrm{~Hz}, 1 \mathrm{H}, \mathrm{CHCH}_{3}\right), 7.38(\mathrm{~s}, 1 \mathrm{H}, \mathrm{CH}) 7.49-7.44(\mathrm{~m}, 3 \mathrm{H}, \mathrm{Ph}-\mathrm{CH}), 7.63-$ $7.57(\mathrm{~m}, 2 \mathrm{H}, \mathrm{Ph}-\mathrm{CH}), 10.01$ (s, 1H, CHO). ${ }^{13} \mathrm{C}$ NMR (100 MHz, DMSO-d $\left.d_{6}\right) \delta: 15.2,21.6,63.6$, 81.9, 86.1, 90.3, 117.7, 121.8, 129.3, 129.8, 131.9, 134.4, 141.0, 181.8. MS, $m / z$ (\%) 268 (17), 239 (10), 196 (100), 139 (18), 73 (34), 45 (67). HRMS (ES) calculated for $\left[\mathrm{M}+\mathrm{Na}^{+} \mathrm{C}_{16} \mathrm{H}_{16} \mathrm{~N}_{2} \mathrm{NaO}_{2}\right.$ : 291.1104; found 291.1103.

4-Bromo-1-(1-ethoxyethyl)-3-(phenylethynyl)-1H-pyrazole (23). 4-Bromo-1-(1-ethoxyethyl)-3iodo- $1 \mathrm{H}$-pyrazole (7a) was used, purification by column chromatography (ethyl acetate : n-hexane $(1: 8), \mathrm{Rf}=0.4)$ gave titled compound as slightly yellow oil, yield $64 \%, 0.204 \mathrm{~g} .{ }^{1} \mathrm{H}$ NMR (400 MHz, DMSO- $\left.d_{6}\right) \delta: 1.06\left(\mathrm{t}, J 7.0 \mathrm{~Hz}, 3 \mathrm{H}, \mathrm{CH}_{2} \mathrm{CH}_{3}\right), 1.60\left(\mathrm{~d}, J 6.0 \mathrm{~Hz}, 3 \mathrm{H}, \mathrm{CHCH}_{3}\right), 3.24$ (dq, $J$ 9.5, 7.0 Hz, 1H, $\left.\mathrm{CHHCH}_{3}\right), 3.46\left(\mathrm{dq}, J 9.5,7.0 \mathrm{~Hz}, 1 \mathrm{H}, \mathrm{CHHCH}_{3}\right), 5.55$ (q, J 6.0 Hz, $1 \mathrm{H}, \mathrm{CHCH}_{3}$ ), 7.50 - 7.42 (m, 3H, Ph-CH), $7.64-7.53$ (m, 2H, Ph-CH), 8.35 (s, 1H, ArH) ${ }^{13} \mathrm{C}$ NMR (100 MHz, DMSO- $\left.d_{6}\right) \delta: 15.1,21.4,64.0,80.6,88.1,92.8,97.8,121.8,129.4,129.8,130.1,131.9,134.4$. MS, $\mathrm{m} / \mathrm{z}$ (\%) $320: 318$ (16 : 16), 248 (94), 246 (100), 194 (7), 139 (16), 73 (30), 45 (64). HRMS (ES) calculated for $[\mathrm{M}+\mathrm{Na}]^{+} \mathrm{C}_{15} \mathrm{H}_{15} \mathrm{BrN}_{2} \mathrm{NaO}$ : 341.0260; found 341.0257 .

1-(1-Ethoxyethyl)-3-(phenylethynyl)-5-(trifluoromethyl)-1H-pyrazole (24). 1-(1-Ethoxyethyl)3-iodo-5-(trifluoromethyl)-1H-pyrazole (8a) was used, purification by column chromatography (ethyl acetate : n-hexane $(1: 20), \mathrm{Rf}=0.5)$ gave titled compound as slightly yellow oil, yield $73 \%$, 0.225 g. ${ }^{1} \mathrm{H}$ NMR (400 MHz, DMSO- $d_{6}$ ) $\delta: 1.09$ (t, $\left.J 7.0 \mathrm{~Hz}, 3 \mathrm{H}, \mathrm{CH}_{2} \mathrm{CH}_{3}\right), 1.63$ (d, $J 5.9 \mathrm{~Hz}, 3 \mathrm{H}$, $\left.\mathrm{CHCH}_{3}\right), 3.25\left(\mathrm{dq}, J\right.$ 9.2, $\left.7.0 \mathrm{~Hz}, 1 \mathrm{H}, \mathrm{CHHCH}_{3}\right), 3.52-3.42\left(\mathrm{~m}, 1 \mathrm{H}, \mathrm{CHHCH}_{3}\right), 5.76(\mathrm{q}, J 5.9 \mathrm{~Hz}$, $\left.1 \mathrm{H}, \mathrm{CHCH}_{3}\right), 7.29(\mathrm{~s}, 1 \mathrm{H}, \mathrm{CH}) 7.54-7.42(\mathrm{~m}, 3 \mathrm{H}, \mathrm{Ph}-\mathrm{CH}), 7.62-7.56(\mathrm{~m}, 2 \mathrm{H}, \mathrm{Ph}-\mathrm{CH}) .{ }^{13} \mathrm{C} \mathrm{NMR}$ $\left(100 \mathrm{MHz}, \mathrm{DMSO}-d_{6}\right) \delta: 15.0,21.7,64.0,81.5,87.6,90.7,112.6,121.6,129.36,129.44,129.9$, 131.95, 131.99, 134.3. MS, m/z (\%) 308 (15), 264 (22), 236 (100), 188 (9), 73 (57), 45 (89). HRMS (ES) calculated for $[\mathrm{M}+\mathrm{Na}]^{+} \mathrm{C}_{16} \mathrm{H}_{15} \mathrm{~F}_{3} \mathrm{~N}_{2} \mathrm{NaO}$ : 331.1029; found 331.1030.

1-(1-Ethoxyethyl)-4-methyl-3-(phenylethynyl)-1H-pyrazole (25). 1-(1-Ethoxyethyl)-3-iodo-4methyl-1H-pyrazole (12a) was used, purification by column chromatography (ethyl acetate : nhexane $(1: 6), \mathrm{Rf}=0.4$ ) gave titled compound as slightly yellow oil, yield $69.7 \%, 0.177 \mathrm{~g} .{ }^{1} \mathrm{H}$ NMR (400 MHz, DMSO- $d_{6}$ ) $\delta: 1.05\left(\mathrm{t}, J 7.0 \mathrm{~Hz}, 3 \mathrm{H}, \mathrm{CH}_{2} \mathrm{CH}_{3}\right), 1.57$ (d, $J 6.0 \mathrm{~Hz}, 3 \mathrm{H}, \mathrm{CHCH}_{3}$ ), $2.11\left(\mathrm{~d}, J 0.7 \mathrm{~Hz}, 3 \mathrm{H}, \mathrm{CH}_{3}\right), 3.20\left(\mathrm{dq}, J 9.6,7.0 \mathrm{~Hz}, 1 \mathrm{H}, \mathrm{CHHCH}_{3}\right), 3.42(\mathrm{dq}, J 9.6,7.1 \mathrm{~Hz}, 1 \mathrm{H}$, $\left.\mathrm{CHHCH}_{3}\right), 5.49$ (q, J 6.0 Hz, 1H, $\left.\mathrm{CHCH}_{3}\right), 7.46-7.41(\mathrm{~m}, 3 \mathrm{H}, \mathrm{Ar}-\mathrm{CH}), 7.59-7.53$ (m, 2H, Ar$\mathrm{CH}) .7 .81(\mathrm{~s}, 1 \mathrm{H}, \mathrm{Ar}-\mathrm{CH}) .{ }^{13} \mathrm{C}$ NMR (101 MHz, DMSO- $\left.d_{6}\right) \delta$ 9.1, 15.2, 21.6, 63.6, 82.5, 87.0, 91.9, 
119.9, 122.6, 127.6, 129.3, 129.3, 131.7, 134.0. MS, m/z (\%) 254 (21), 210 (20), 182 (100), 128 (7), 77 (4), 73 (9), 45 (30). HRMS (ES) calculated for [M+Na] ${ }^{+} \mathrm{C}_{16} \mathrm{H}_{18} \mathrm{~N}_{2} \mathrm{NaO}$ : 277.1311; found 277.1312 .

1-(1-Ethoxyethyl)-3-(phenylethynyl)-1H-pyrazole-4-carbaldehyde (26). 1-(1-Ethoxyethyl)-3iodo- $1 H$-pyrazole-4-carbaldehyde (17) was used, purification by column chromatography (ethyl acetate : $\mathrm{n}$-hexane $(1: 4), \mathrm{Rf}=0.4)$ gave titled compound as slightly yellow crystals, yield $86.2 \%$, $0.231 \mathrm{~g}, \mathrm{mp} 72-74{ }^{\circ} \mathrm{C} .{ }^{1} \mathrm{H}$ NMR (400 MHz, DMSO- $\left.d_{6}\right) \delta: 1.08$ (t, $\left.J 7.0 \mathrm{~Hz}, 3 \mathrm{H}, \mathrm{CH}_{2} \mathrm{CH}_{3}\right), 1.63$ (d, $\left.J 5.9 \mathrm{~Hz}, 3 \mathrm{H}, \mathrm{CHCH}_{3}\right), 3.28\left(\mathrm{dq}, J 9.5,7.0 \mathrm{~Hz}, 1 \mathrm{H}, \mathrm{CHHCH}_{3}\right), 3.50(\mathrm{dq}, J 9.5,7.0 \mathrm{~Hz}, 1 \mathrm{H}$, $\left.\mathrm{CHHCH}_{3}\right), 5.67$ (q, J $\left.5.9 \mathrm{~Hz}, 1 \mathrm{H}, \mathrm{CHCH}_{3}\right), 7.50-7.45$ (m, 3H, Ph-CH), 7.66 - 7.62 (m, 2H, Ph$\mathrm{CH}), 8.75$ (s, 1H, ArH), 9.95 (s, 1H, CHO). ${ }^{13} \mathrm{C}$ NMR (100 MHz, DMSO- $\left.d_{6}\right) \delta: 15.1,21.5,64.2$, 80.8, 88.0, 93.1, 121.7, 124.7, 129.3, 130.0, 132.1, 133.8, 135.1, 184.6. MS, $\mathrm{m} / z$ (\%) 268 (25), 239 (5), 209 (81), 196 (65), 195 (65), 139 (15), 73 (52), 45 (100). HRMS (ES) calculated for [M+Na] $]^{+}$ $\mathrm{C}_{16} \mathrm{H}_{16} \mathrm{~N}_{2} \mathrm{NaO}_{2}$ : 291.1104; found 291.1105.

1-Methyl-3-(phenylethynyl)-1H-pyrazole (27). 3-Iodo-1-methyl-1H-pyrazole (18) was used, purification by column chromatography (ethyl acetate : n-hexane $(1: 4), \mathrm{Rf}=0.2)$ gave titled compound as slightly yellow oil, yield 82\%, $0.15 \mathrm{~g} .{ }^{1} \mathrm{H}$ NMR $\left(400 \mathrm{MHz}, \mathrm{DMSO}-d_{6}\right) \delta: 3.87(\mathrm{~s}, 3 \mathrm{H}$, $\left.\mathrm{CH}_{3}\right), 6.52(\mathrm{~d}, J 2.2 \mathrm{~Hz}, 1 \mathrm{H}, \mathrm{CH}), 7.45-7.40$ (m, 3H, Ph-CH), $7.56-7.51$ (m, 2H, Ph-CH), 7.78 $(\mathrm{d}, J 2.2 \mathrm{~Hz}, 1 \mathrm{H}, \mathrm{CH}) .{ }^{13} \mathrm{C}$ NMR $\left(100 \mathrm{MHz}, \mathrm{DMSO}-d_{6}\right) \delta: 39.4,83.7,89.1,110.0,122.6,129.3$, 131.7, 132.2, 133.4, 133.8. MS, m/z (\%) 182 (100), 154 (16), 127 (18), 91 (22), 42 (13). HRMS (ES) calculated for $[\mathrm{M}+\mathrm{Na}]^{+} \mathrm{C}_{12} \mathrm{H}_{10} \mathrm{~N}_{2} \mathrm{Na}$ : 205.0736; found 205.0734.

1-(1-Ethoxyethyl)-3-nitro-4-(phenylethynyl)-1H-pyrazole (28). 1-(1-Ethoxyethyl)-4-iodo-3nitro-1 $H$-pyrazole (10a) was used, purification by recrystallization from $\mathrm{n}$-hexane gave titled compound as slightly yellow crystals, yield $58 \%, 0.165 \mathrm{~g}, \mathrm{mp} 108-111^{\circ} \mathrm{C} .{ }^{1} \mathrm{H}$ NMR $(400 \mathrm{MHz}$, DMSO- $\left.d_{6}\right) \delta: 1.10\left(\mathrm{t}, J 7.0 \mathrm{~Hz}, 3 \mathrm{H}, \mathrm{CH}_{2} \mathrm{CH}_{3}\right), 1.64\left(\mathrm{~d}, J 5.9 \mathrm{~Hz}, 3 \mathrm{H}, \mathrm{CHCH}_{3}\right), 3.38-3.28(\mathrm{~m}, 1 \mathrm{H}$, $\left.\mathrm{CHHCH}_{3}\right), 3.53\left(\mathrm{dq}, J\right.$ 9.5, 7.0 Hz, 1H, $\left.\mathrm{CHHCH}_{3}\right), 5.70$ (q, J $\left.5.9 \mathrm{~Hz}, 1 \mathrm{H}, \mathrm{CHCH}_{3}\right), 7.49-7.45(\mathrm{~m}$, $3 \mathrm{H}, \mathrm{Ph}-\mathrm{CH}), 7.58-7.53(\mathrm{~m}, 2 \mathrm{H}, \mathrm{Ph}-\mathrm{CH}), 8.70(\mathrm{~s}, 1 \mathrm{H}, \mathrm{CH}) .{ }^{13} \mathrm{C}$ NMR $\left(100 \mathrm{MHz}\right.$, DMSO- $\left.d_{6}\right) \delta$ : 15.1, 21.5, 64.6, 78.5, 89.4, 93.8, 100.1, 122.2, 129.36, 129.38, 129.8, 131.8, 135.6. MS, $\mathrm{m} / \mathrm{z}(\%)$ 285 (22), 240 (6), 213 (57), 105 (94), 73 (52), 45 (100). HRMS (ES) calculated for [M+Na] $\mathrm{C}_{15} \mathrm{H}_{15} \mathrm{~N}_{3} \mathrm{NaO}_{3}$ : 308.1006; found 308.1011.

\section{References}

1. Stauffer, S. R.; Huang, Y. R.; Aron, Z. D.; Coletta, C. J.; Sun, J.; Katzenellenbogen, B. S.; Katzenellenbogen, J. A. Bioorganic \& Medicinal Chemistry 2001, 9, 151. http://dx.doi.org/10.1016/S0968-0896(00)00226-1

2. Kumar, V.; Kaur, K.; Gupta, G. K.; Sharm,a A. K. European Journal of Medicinal Chemistry 2013, 69, 735 .

http://dx.doi.org/10.1016/j.ejmech.2013.08.053 
3. Sasabe, H.; Kido, J. Chem. Mater. 2011, 23, 621. http://dx.doi.org/10.1021/cm1024052

4. Ojwach, S. O.; Darkwa, J. Inorg. Chim. Acta 2010, 363, 1947. http://dx.doi.org/10.1016/j.ica.2010.02.014

5. Vasilevsky, S. F.; Mshvidobadze, E. V.; Mamatyuk, V. I.; Romanenkoc, G. V.; Elguerod, J. Tetrahedron Letters 2005, 46, 4457. http://dx.doi.org/10.1016/j.tetlet.2005.04.127

6. Zhang, J.; Zhang, Y.; Schnatter, W. F. K.; Herndon, J. W. Organometallics 2006, 25, 1279. http://dx.doi.org/10.1021/om051008q

7. Heller, S. T.; Natarajan, S. R. Org. Lett. 2007, 9, 4947. http://dx.doi.org/10.1021/ol701784w

8. Fustero, S.; Sanchez-Rosello, M.; Barrio, P.; Simon-Fuentes, A. Chem. Rev. 2011, 111, 6984. http://dx.doi.org/10.1021/cr2000459

9. Cottineau, B.; Cenault, J. Synlett. 2002, 769. http://dx.doi.org/10.1055/s-2002-25331

10. Delaunay, T.; Genix, P.; Es-Sayed, M.; Vors, J. P.; Monteiro, N.; Balme, G. Org. Lett. 2010, 12,3328 . http://dx.doi.org/10.1021/ol101087j

11. Zhang, T.; Gao, X.; Wood, H. B. Tetrahedron Letters 2011, 52, 311. http://dx.doi.org/10.1016/j.tetlet.2010.11.037

12. Cikotiene, I.; Buksnaitiene, R.; Sazinas, R. Tetrahedron 2011, 67, 706. http://dx.doi.org/10.1016/j.tet.2010.11.073

13. Ouyang, H. C.; Tang, R. Y.; Zhong, P.; Zhang X. G.; Li J. H. J. Org. Chem. 2011, 76, 223. http://dx.doi.org/10.1021/jo102060j

14. Barluenga, J.; Vazquez-Villa, H.; Ballesteros, A.; Gonzalez, J. M. J. Am. Chem. Soc. 2003, 125, 9028. http://dx.doi.org/10.1021/ja0355372

15. Tao, X.; Guosheng, L. Org. Lett. 2012, 14, 5416. http://dx.doi.org/10.1021/ol3026507

16. Cikotiene, I.; Sazinas, R.; Mazeikaite, R.; Labanauskas, L. Synlett. 2010, $20,3027$. http://dx.doi.org/10.1055/s-0030-1259053

17. Taydakov, I. V.; Krasnoselskiy, S. S.; Dutova, T. Y. Synthetic Communications, 2011, 41, 2430. http://dx.doi.org/10.1080/00397911.2010.503002

18. Lin, Q.; Meloni, D.; Pan, Y.; Xia, M.; Rodgers, J.; Shepard, S.; Li, M.; Galya, L.; Metcalf, B.; Yue, T. Y.; Liu, P.; Zhou, J. Org. Lett. 2009, 11, 1999. http://dx.doi.org/10.1021/o1900350k

19. Vasilevsky, S. F.; Klyatskaya, S. V.; Tretyakova, E. V.; Elguero, J. Heterocycles 2003, 60, 879. http://dx.doi.org/10.3987/COM-02-9698 
20. McLaughlin, M.; Marcantonio, K.; Chen, C.; Davies, I. W. J. Org. Chem. 2008, 73, 4309. http://dx.doi.org/10.1021/jo800321p

21. Primas, N.; Bouillon, A.; Rault, S. Tetrahedron 2010, 66, 8121. http://dx.doi.org/10.1016/j.tet.2010.08.001

22. Ivachtchenko, A. V.; Kravchenko, D. V.; Zheludeva, V. I.; Pershin, D. G. Journal of Heterocyclic Chemistry 2004, 41, 931. http://dx.doi.org/10.1002/jhet.5570410612

23. Kashima, C.; Tsurouka, S.; Mizuhara, S. Tetrahedron 1998, 54, 14679.

http://dx.doi.org/10.1016/S0040-4020(98)00947-8

24. Trofimenko, S. J. Am. Chem. Soc. 1970, 92, 5118.

http://dx.doi.org/10.1021/ja00720a021 